\title{
STABILITY PROPERTIES OF SOLITARY WAVES FOR FRACTIONAL KDV AND BBM EQUATIONS
}

\author{
JAIME ANGULO PAVA \\ Department of Mathematics, IME-USP \\ Rua do Matão 1010, Cidade Universitária, CEP 05508-090, São Paulo, SP, Brazil. \\ angulo@ime.usp.br
}

\begin{abstract}
This paper sheds new light on the stability properties of solitary wave solutions associated with models of Korteweg-de Vries and Benjamin\&Bona\&Mahoney type, when the dispersion is very lower. Via an approach of compactness, analyticity and asymptotic perturbation theory, we establish sufficient conditions for the existence of exponentially growing solutions to the linearized problem and so a criterium of linear instability of solitary waves is obtained for both models. Moreover, the nonlinear stability and linear instability of the ground states solutions for both models is obtained for some specific regimen of parameters. Via a Lyapunov strategy and a variational analysis we obtain the stability of the blow-up of solitary waves for the critical fractional KdV equation.

The arguments presented in this investigation has prospects for the study of the instability of traveling waves solutions of other nonlinear evolution equations.
\end{abstract}

Keywords: Orbital stability, Linear instability, Lower dispersion models, fKdV equations, fBBM equations.

Mathematical subject classification: 76B25, 35Q51, 35Q53.

Date: January 20, 2017

\section{INTRODUCTION}

This paper provides a detailed study of various stability issues associated to the dynamic of solitary waves solutions for the so-called fractional Korteweg-de Vries equation (henceforth fKdV equation)

$$
u_{t}+u^{p} u_{x}-D^{\alpha} u_{x}=0, \quad p \in \mathbb{N},
$$

where $u=u(x, t), x, t \in \mathbb{R}$, represents a real valued function, and $D^{\alpha}$ is defined via Fourier transform by

$$
\widehat{D^{\alpha}} f(\xi)=|\xi|^{\alpha} \widehat{f}(\xi), \quad \alpha \in(0,1) .
$$

The importance of study of this model for any $\alpha>0$ is due to its physical relevance and its own mathematical interest. We recall that the model (1.1) contains two famous family of equations, the generalized Korteweg-de Vries for $\alpha=2$ (gKdV henceforth), and 
the generalized Benjamin-Ono equation for $\alpha=1$ (gBO henceforth), and in this case $D$ can be write as $D=\mathcal{H} \partial_{x}$, where $\mathcal{H}$ denotes the Hilbert transform and which may be defined by $\widehat{\mathcal{H} f}(\xi)=-i \operatorname{sgn}(\xi) \widehat{f}(\xi)$.

For $\alpha \geqq 1$, studies on the Cauchy problem, blow-up issues, large-time asymptotic behavior of solutions, the stability of solitary waves solutions, breathers solutions and multi-solitons solutions (as well as periodic traveling wave solutions) have been the focus of deep research in the past years via a rich variety of techniques, see by instance, Albert [1], Albert\&Bona [2], Albert\&Bona\&Saut [3], Alejo\&Muñoz [4], Angulo [5], Angulo\&Bona\&Scialom [7], Benjamin [9]-[10], Bona [12], Bona\&Souganidis\&Strauss [14], Bona\&Saut [13], Iorio [24], Grillakis\&Shatah\&Strauss [19]-[20], Kenig\&Ponce\&Vega [28], Lopes [37], Martel\&Merle [39]-40]-[41], Martel\&Pilod [42], Muñoz [43], Weinstein [50][51].

The case $\alpha \in(0,1)$ has been the focus of many recent studies. The Cauchy problem, the existence of solitary wave solutions, the stability properties of the ground states and numerical simulations have been addressed by Linares\&Pilod\&Saut [35]-[36], Frank\&Lenzmann [16] and Klein\&Saut [29].

One of the objectives of this paper is to extend the theory of Vock\&Hunziker in [48] about the stability of Schrödinger eigenvalue problems to the study of linear instability of solitary waves solutions for the fKdV equation with a "lower dispersion" (see Theorems 1.2, 1.3 below). In particular, we recover the linear instability results in [25] for the ground state solutions (see Definition 1.1 below) of (1.1) with $p=1$ and $\alpha \in\left(\frac{1}{3}, \frac{1}{2}\right)$. For completeness of the exposition, we show in an unified way the nonlinear stability results for the ground state solutions with $p<2 \alpha$ and $\alpha \in\left(\frac{1}{2}, 2\right)$ (see Theorem 1.1 below).

The case $\alpha=\frac{1}{2}$ and $p=1$ in (1.1), so-called the critical case for the fKdV model, it remains open for a stability analysis of solitary waves. Indeed, in this case, the recently numerical simulations in Klein\&Saut 29] suggest the existence of blow up of solutions for initial data close to the solitary waves and proving such result seems to be out of reach. Here, we will show that for this critical case a kind of "stability of the blow-up" near to the possible unstable ground state solutions happens and we checked one of the conjectures emerging of the numerical findings in [29] (see Theorem 3.1 below).

Our approach of linear instability for the solitary wave solutions of (1.1) is extended to the following generalized fractional Korteweg-de Vries models (gfKdV henceforth)

$$
u_{t}+(f(u))_{x}-(\mathcal{M} u)_{x}=0
$$

where $\mathcal{M}$ is a differential or pseudo-differential operator defined as a Fourier multiplier operator

$$
\widehat{\mathcal{M} g}(\xi)=\beta(\xi) \widehat{g}(\xi), \quad \xi \in \mathbb{R},
$$

and, $f$ is assumed to be a smooth nonlinear function. The symbol $\beta$ of $\mathcal{M}$ (representing the lower dispersion effects) is assumed to be continuous, locally bounded, even function 
on $\mathbb{R}$, satisfying the conditions

$$
a_{1}|\xi|^{\gamma} \leq \beta(\xi) \leq a_{2}(1+|\xi|)^{\alpha},
$$

for $|\xi| \geq b_{0}, 0 \leq \gamma \leqq \alpha<1$, with $\beta(\xi)>b$, for all $\xi \in \mathbb{R}$ and $a_{i}>0, i=1,2$. In this point of the analysis, we extend the linear instability results in Lin 34 for the models (1.2) with a growth of the symbol of $\mathcal{M}$ determined by $\alpha \in(0,1)$.

We note, that in various models of fluid dynamics and mathematical physics the symbol $\beta$ in (1.3) is not necessarily polynomial, such as in the case of the Whitham equation for describing water waves in the small amplitude and long wave regime when surface tension is included ([32], [33], [49])

$$
\beta(\xi)=\left(1+\gamma|\xi|^{2}\right)^{1 / 2}\left(\frac{\tanh \xi}{\xi}\right)^{1 / 2}
$$

where $\gamma \geqq 0$ measures the surface tension effects. Here $\beta$ satisfies

$$
\frac{1}{2}|\xi|^{1 / 2} \leqq \beta(\xi) \leqq 2|\xi|^{1 / 2}, \text { for }|\xi| \text { large. }
$$

The analysis established above for the fKdV equation (1.1) was also extended to the fractional $\mathrm{BBM}$ equation (fBBM henceforth)

$$
u_{t}+u_{x}+\partial_{x}\left(u^{2}\right)+D^{\alpha} u_{t}=0,
$$

for $\alpha \in\left(\frac{1}{3}, 1\right)$. In this case we show that the ground state solutions associated to the fBBM equation are linearly unstable for $\alpha \in\left(\frac{1}{3}, \frac{1}{2}\right)$ with a wave-speed not so large. For $\alpha \in\left(\frac{1}{3}, 1\right)$ we have also nonlinear stability in the case of the wave-speed in general to be large (see Theorems 1.4 1.5 below).

Before of establishing more precisely our results, we will make a brief summary of some known results for the fKdV model, $\alpha \in(0,1)$, it which will be useful in our exposition. We start initially with some basic information about the existence of solitary waves solutions for this model. A solitary wave solution for (1.1) is a solution of the form $u(x, t)=$ $\varphi_{c}(x-c t)$ with

$$
\lim _{|\xi| \rightarrow \infty} \varphi_{c}(\xi)=0
$$

which (if they exist !!!) it will represent a "perfect" balance between the lower dispersion and the effects of the nonlinearity. For $\varphi \equiv \varphi_{c}$ belongs to the space $H^{\alpha / 2}(\mathbb{R}) \cap L^{p+2}(\mathbb{R})$ we have that

$$
D^{\alpha} \varphi+c \varphi-\frac{1}{p+1} \varphi^{p+1}=0 .
$$

The existence of solutions for (1.6), with the later specify regularity conditions, it can be deduced from the Concentration-Compactness Method for any $c>0$ and $p \in\left(1, \frac{2 \alpha}{1-\alpha}\right)$ (see Weinstein [51] and Arnesen [8]). We can also to see (by the so-called Pohozaev identities) that the pseudo-differential equation satisfied by the profile $\varphi$ does not admit any non-trivial solutions for the following cases: 
(1) for $\alpha \geqq 1$ and $c<0$ (without restrictions on the power $p$ ),

(2) for $\alpha \in(0,1), c>0$ and $\alpha \leqq \frac{p}{p+2}$.

For completeness of the exposition, we will establish the item (2) above (the item (1) is very well known). Indeed, for $\alpha \in(0,1)$ we have from Lemma B.2 in Frank\&Lenzmann [16] that $\varphi \in H^{\alpha+1}(\mathbb{R})$. Then by Plancherel Theorem, the following energy identity is immediate

$$
\int_{\mathbb{R}}\left|D^{\alpha / 2} \varphi\right|^{2} d x+c \int_{\mathbb{R}} \varphi^{2} d x-\frac{1}{p+1} \int_{\mathbb{R}} \varphi^{p+2} d x=0 .
$$

Next, since $\varphi^{\prime}$ makes sense, we have by Plancherel and integration by parts that

$$
\int_{\mathbb{R}} x \varphi^{\prime} D^{\alpha} \varphi d x=\frac{\alpha-1}{2} \int_{\mathbb{R}}\left|D^{\alpha / 2} \varphi\right|^{2} d x .
$$

Thus, from (1.7)-(1.8) follows

$$
(\alpha(p+2)-p) \int_{\mathbb{R}}\left|D^{\alpha / 2} \varphi\right|^{2} d x=c p \int_{\mathbb{R}}|\varphi|^{2} d x
$$

proving that no finite energy solitary waves exist when $c>0$ and $\alpha \leqq \frac{p}{p+2}$ hold.

The following definition will be useful in our study (see Frank\&Lenzmann [16]).

Definition 1.1. Let $Q \in H^{\alpha / 2}(\mathbb{R})$ be an even and positive solution of

$$
D^{\alpha} Q+Q-Q^{p+1}=0 \quad \text { in } \mathbb{R} .
$$

If $Q$ solves the minimization problem

$$
J^{\alpha, p}(Q)=\inf \left\{J^{\alpha, p}(v): v \in H^{\alpha / 2}(\mathbb{R})-\{0\}\right\}
$$

where $J^{\alpha, p}$ is the 'Weinstein' functional

$$
J^{\alpha, p}(v)=\frac{\left(\int_{\mathbb{R}}\left|D^{\frac{\alpha}{2}} v\right|^{2}\right)^{\frac{p}{2 \alpha}}\left(\int_{\mathbb{R}}|v|^{2}\right)^{\frac{p}{2 \alpha}(\alpha-1)+1}}{\int_{\mathbb{R}}|v|^{p+2}},
$$

then we say that $Q \in H^{\alpha / 2}(\mathbb{R})$ is a ground state solution of equation (1.10). Here, $0<\alpha<2$ and $0<p<p_{\max }(\alpha)$, and where the critical exponent $p_{\max }(\alpha)$ is defined as

$$
p_{\text {max }}(\alpha) \equiv \begin{cases}\frac{2 \alpha}{1-\alpha}, & \text { for } 0<\alpha<1 \\ +\infty, & \text { for } 1 \leqq \alpha<2 .\end{cases}
$$

From Frank\&Lenzmann (Proposition 1.1 and Theorem 2.2 in [16]) there is a unique (modulo translation) ground state solution for (1.10). For $p<p_{\max }(\alpha)$ and $\alpha \in(0,1)$, it is so-called the case $H^{\alpha / 2}$-subcritical, because of this condition on $p$ is necessary to have the existence of solutions for (1.10) (see the analysis above). Thus, via a scaling argument, we 
obtain that equation in (1.6) has a unique ground state solution, denoted by $Q_{c}$. Moreover, we have the following regularity and decay properties for $Q_{c}: Q_{c} \in H^{\alpha+1}(\mathbb{R}) \cap C^{\infty}(\mathbb{R})$,

$$
\frac{C_{0}}{1+|x|^{\alpha+1}} \leqq\left|Q_{c}(x)\right| \leqq \frac{C}{1+|x|^{\alpha+1}}, \quad\left|x Q_{c}^{\prime}(x)\right| \leqq \frac{C}{1+|x|^{\alpha+1}}, \quad \text { for all } x \in \mathbb{R},
$$

with some constants $C \geqq C_{0}>0$ depending of $\alpha, p$ and $c$.

The study of stability properties for the solitary wave profile $\varphi$ in (1.6) for the case $\alpha \geqq 1$ is well developed. Indeed, in few words, there are two useful lines of exploration for studying this relevant property in the vicinity of the wave $\varphi$. First, we have a global variational characterization of solutions of (1.6) such that a profile $\varphi$ satisfying that $\varphi>0$ on $\mathbb{R}, \varphi$ even and $\varphi^{\prime}<0$ on $(0,+\infty)$ can be seen as the infima of the constrained-mass energy minimizer

$$
J=\inf \left\{E(v): v \in H^{\alpha / 2}(\mathbb{R}) \text { and } \int_{\mathbb{R}} v^{2} d x=\lambda\right\}
$$

with $E$ being the conservation-energy functional

$$
E(v)=\frac{1}{2} \int_{\mathbb{R}}\left|D^{\alpha / 2} v\right|^{2}-\frac{2}{(p+1)(p+2)} v^{p+2} d x .
$$

We recall, since $\alpha \geqq 1$, the Sobolev embedding $H^{\alpha / 2}(\mathbb{R}) \hookrightarrow L^{p+2}(\mathbb{R})$ ensures that the functional $E$ is well-defined for any $p \geqq 0$ and the infimum in (1.15) will satisfy $-\infty<$ $J<0$ exactly for $p<2 \alpha$ (the so-called $L^{2}$-subcritical case). Thus, the ConcentrationCompactness Method will work very well for obtaining both existence and stability properties of $\varphi$. More exactly, in this case we obtain the global stability property of the nonempty set of minimizer $\mathcal{G}$ associated to the variational problem (1.15),

$$
\mathcal{G}=\left\{v \in H^{\alpha / 2}(\mathbb{R}): E(v)=J \text { and } \int_{\mathbb{R}} v^{2} d x=\lambda\right\} .
$$

Thus, via a scaling argument and from the uniqueness results of the ground state solutions $Q_{c}$ of (1.6) for $1 \leqq \alpha \leqq 2$ (see Remark 2.1 in [16]), we obtain for a specific choice of $\lambda$ in (1.17) that

$$
\mathcal{G}=\left\{Q_{c}(\cdot+y): y \in \mathbb{R}\right\} \equiv \Omega_{Q_{c}},
$$

where $\Omega_{Q_{c}}$ is called the orbit generated by $Q_{c}$ via the basic symmetry of translations associated to the model (1.1). For the case $p \geqq 2 \alpha$, it is well known that the profile $\varphi$ is nonlinearly unstable (see Bona\&Souganidis\&Strauss [14] for the case $p>2 \alpha(\alpha \geqq 1)$, Martel\&Merle [40]- [41] for $\alpha=2, p=4$, and Merle\&Pilod [42] for $\alpha=1, p=2$ ). We note that by using a variational approach, it is also possible to obtain the instability result in the $L^{2}$-supercritical case $p>2 \alpha \geqq 2$ (see Angulo's book, Chapter 10, [5])

An similar approach of stability for the orbit $\Omega_{Q_{c}}$ in the case $\frac{1}{2}<\alpha<1$ and $p<2 \alpha(p=$ 1), it has been established recently by Linares\&Pilod\&Saut in [35]. The ConcentrationCompactness Method was applied successfully to the minimizer problem in (1.15) and again the property $-\infty<J<0$ is necessary for the stability result. In this point, it is 
also worth noting that for $\alpha=1 / 2, J=0$. Indeed, since the ground state for (1.6) with $\alpha>1 / 3$ is characterized (via a scaling) as the solution of the minimization problem $J^{\frac{1}{2}, 1}$ in (1.11), we obtain from $J^{\frac{1}{2}, 1}\left(Q_{c}\right)=\frac{1}{3}\left\|Q_{c}\right\|$ the sharp inequality

$$
\frac{1}{3} \int_{\mathbb{R}}|v|^{3} d x \leqq \frac{\|v\|}{\left\|Q_{c}\right\|} \int_{\mathbb{R}}\left|D^{\frac{1}{4}} v\right|^{2} d x .
$$

Thus, for the restriction $\|v\|=\left\|Q_{c}\right\|$ we obtain immediately that $E(v) \geqq 0$ and $E\left(Q_{c}\right)=0$. Moreover, from (1.19) it follows the following main property:

$$
\text { if }\|v\| \leqq\left\|Q_{c}\right\| \quad \text { then } \quad E(v) \geqq 0 .
$$

We recall that the later result is similar to that for $\alpha \geqq 1$ and $2 \alpha=p$, namely, $J^{\alpha, 2 \alpha}\left(Q_{c}\right)=0$, the so-called $L^{2}$-critical case (we note that, for $\alpha \in[1,2]$ and $|u|^{2 \alpha} u_{x}$ as the nonlinear part in (1.1), recently Kenig\&Martel\&Robbiano in [27] have proved for $\alpha$ close to 2 , solutions of negative energy $E$ close to the ground state blow up in finite or infinite time in the energy space $\left.H^{\frac{\alpha}{2}}(\mathbb{R})\right)$. The case $\alpha=\frac{1}{2}$ is so-called the critical case for the fKdV model (1.1).

From the recently numerical study in Klein\&Saut in [29], the simulations showed a possible blow-up phenomenon of the associated solutions for (1.1) with an initial data $u_{0}$ of negative energy $\left(E\left(u_{0}\right)<0\right)$ and therefore with a mass larger that the ground state mass $Q_{c}\left(\left\|Q_{c}\right\|<\left\|u_{0}\right\|\right)$ (see Fig. 10 in [29]). Here we will show in Theorem 3.1 below, that in fact for this regimen of $\alpha$ we have a kind of "stability of the blow-up" near to the possible unstable ground state solutions and so checking one of the conjectures emerging of the numerical findings in 29 .

The second approach for an analysis of orbital stability is that of local type, more exactly, it is fixed a solitary wave profile $\varphi_{c}$ of (1.6) and we study the behavior of the flow associated to (1.1) in a neighborhood of the orbit $\Omega_{\varphi_{c}}$. The main property of the energy $E$ to be obtained in this case is the following:

$$
\left\{\begin{array}{l}
\text { There are } \delta>0 \text { and } \beta_{0}>0 \text { such that } \\
E(v)-E\left(\varphi_{c}\right) \geqq \beta_{0}\left[d\left(v ; \Omega_{\varphi_{c}}\right)\right]^{2} \\
\text { for } d\left(v ; \Omega_{\varphi_{c}}\right)<\delta \text { and } F(v)=F\left(\varphi_{c}\right),
\end{array}\right.
$$

with $F(v)=\frac{1}{2} \int v^{2} d x$ and $d\left(v ; \Omega_{\varphi_{c}}\right)=\inf _{y \in \mathbb{R}}\left\|v-\varphi_{c}(\cdot+y)\right\|_{H^{\frac{\alpha}{2}}}$. So, from (1.21), the continuity of the functional $E$ and of the flow $t \rightarrow u(t)$, we obtain immediately the stability property of $\Omega_{\varphi_{c}}$ by initial perturbations in the manifold

$$
\mathcal{M}=\left\{v: \int_{\mathbb{R}} v^{2} d x=\int_{\mathbb{R}} \varphi_{c}^{2} d x\right\} .
$$

The stability for general perturbations of $\Omega_{\varphi_{c}}$ can be obtained via the existence of a regular curve of solitary waves, $c \rightarrow \varphi_{c}$. 
Now, a way for obtaining (1.21) is to use Taylor's theorem and so the analysis is reduced to study the quadratic form $\left\langle\mathcal{L}_{c} f, f\right\rangle$ on the tangent space to the manifold $\mathcal{M}$ at the point $\varphi_{c}, T_{\varphi_{c}} \mathcal{M}$. Here $\mathcal{L}_{c}$ represents the second variation of the action $S(v)=E(v)+c F(v)$ at the point $v=\varphi_{c}$, namely, the unbounded self-adjoint operator

$$
S^{\prime}\left(\varphi_{c}\right) \equiv \mathcal{L}_{c}=D^{\alpha}+c-\varphi_{c}^{p}
$$

with domain $\mathcal{D}\left(\mathcal{L}_{c}\right)=H^{\alpha}(\mathbb{R})$. Thus, it is well known that proving the inequality

$$
\left\langle\mathcal{L}_{c} f, f\right\rangle \geqq \beta_{1}\|f\|_{H^{\frac{\alpha}{2}}} \quad \text { for every } f \in T_{\varphi_{c}} \mathcal{M} \cap \operatorname{Ker}\left(\mathcal{L}_{c}\right)^{\perp}
$$

for $\beta_{1}>0$ and $\operatorname{Ker}\left(\mathcal{L}_{c}\right)$ representing the kernel of $\mathcal{L}_{c}$, we obtain the key inequality (1.21). The direct check of condition (1.24) is in general extremely inconvenient, because no requirement is directly related to the number (counting multiplicity) of negative eigenvalue of $\mathcal{L}_{c}$ (it which will be denoted henceforth by $n\left(\mathcal{L}_{c}\right)$, in other words, the Morse index of $\left.\mathcal{L}_{c}\right)$. Moreover, in general this operator has a nontrivial negative eigenspace. Indeed, for $\varphi_{c}$ being a positive solitary wave solution we obtain immediately $\left\langle\mathcal{L}_{c} \varphi_{c}, \varphi_{c}\right\rangle<0$ and so the Mini-Max principle implies $n\left(\mathcal{L}_{c}\right) \geqq 1$. The works in Benjamin [10], Weinstein [50]-[51] and Grillakis\&Shatah\&Strauss [19] finesses this difficulty and provides a nice test that guarantees when (1.24) is satisfied. More exactly, we suppose that $n\left(\mathcal{L}_{c}\right)=1$, $\operatorname{Ker}\left(\mathcal{L}_{c}\right)=\left[\frac{d}{d x} \varphi_{c}\right]$ and the remainder of the spectrum of $\mathcal{L}_{c}$ is positive and bounded away from zero. Then, the strictly increasing property of the mapping $c \rightarrow \int_{\mathbb{R}} \varphi_{c}^{2} d x$ will imply inequality (1.24) and so the stability property of $\Omega_{\varphi_{c}}$ follows from (1.21).

Next, we call the attention about the assumption of the existence of a $C^{1}$-mapping $c \rightarrow \varphi_{c}$ of solitary waves. If we assume this condition hold for every $c>0$ and by considering the new variable

$$
\phi(x)=c^{-\frac{1}{p}} \varphi_{c}\left(c^{-\frac{1}{\alpha}} x\right),
$$

we see that $\phi$ will be a solution of

$$
D^{\alpha} \phi+\phi-\frac{1}{p+1} \phi^{p+1}=0
$$

Note the independence of $\phi$ with regard to the wave-speed $c$. Therefore,

$$
\frac{d}{d c} \int_{\mathbb{R}} \varphi_{c}^{2} d x=\|\phi\|^{2} \frac{d}{d c} c^{\frac{2}{p}-\frac{1}{\alpha}}=\left(\frac{2}{p}-\frac{1}{\alpha}\right) c^{\frac{2}{p}-\frac{1}{\alpha}-1}\|\phi\|^{2} .
$$

Therefore,

$$
\frac{d}{d c} \int_{\mathbb{R}} \varphi_{c}^{2} d x>0 \Leftrightarrow p<2 \alpha .
$$

Thus we see that condition in (1.27) is the same imposed for obtaining a minimum of the variational problem (1.15) at least for $\alpha>\frac{1}{2}$, and therefore it is not a technical condition for the method works !. 
Next, if we consider that the curve $c \rightarrow \varphi_{c}$ has a sufficiently regularity, then differentiating (1.6) with regard to the variable $c$, we obtain that

$$
\mathcal{L}_{c}\left(-\frac{d}{d c} \varphi_{c}\right)=\varphi_{c}
$$

Now, if for some $\psi \in D\left(\mathcal{L}_{c}\right)$ we have that $\mathcal{L}_{c} \psi=\varphi_{c}$, then from (1.28) it follows that

$$
\mathcal{L}_{c}\left(\frac{d}{d c} \varphi_{c}+\psi\right)=0 .
$$

Hence, if we suppose that $\operatorname{Ker}\left(\mathcal{L}_{c}\right)=\left[\frac{d}{d x} \varphi_{c}\right]$ then $\frac{d}{d c} \varphi_{c}+\psi=\theta \frac{d}{d x} \varphi_{c}$, for $\theta \in \mathbb{R}$, and therefore

$$
\left\langle\psi, \varphi_{c}\right\rangle=-\frac{1}{2} \frac{d}{d c} \int_{\mathbb{R}} \varphi_{c}^{2} d x
$$

So, we have that the condition of strictly increasing of the mapping $c \rightarrow \int_{\mathbb{R}} \varphi_{c}^{2} d x$ can be replaced by the condition:

$$
\text { if } \mathcal{L}_{c} \psi=\varphi_{c}, \quad \text { then }\left\langle\psi, \varphi_{c}\right\rangle=\left\langle\mathcal{L}_{c}^{-1} \varphi_{c}, \varphi_{c}\right\rangle<0
$$

Condition (1.29) is useful in situations where it is not clear the existence of a family of solitary waves $\varphi_{c}$ depending smoothly on $c$ (see Albert [1]). We recall that as $\mathcal{L}_{c}$ is a self-adjoint operator and $\varphi_{c} \in \operatorname{Ker}\left(\mathcal{L}_{c}\right)^{\perp}$, the Fredholm solvability theorem guarantees always the existence of an element $\psi \in D\left(\mathcal{L}_{c}\right)$ such that $\mathcal{L}_{c} \psi=\varphi_{c}$.

Before establishing our first stability result, let us to define orbital stability for equation (1.1). If $\varphi$ is a given solitary wave solution of (1.6); define for any $\eta>0$ the set $U_{\eta} \subset$ $H^{\frac{\alpha}{2}}(\mathbb{R})$ by

$$
U_{\eta}=\left\{v \in H^{\frac{\alpha}{2}}: i n f_{y \in \mathbb{R}}\|v-\varphi(\cdot+y)\|_{H^{\frac{\alpha}{2}}}<\eta\right\}
$$

Definition 1.2. $\varphi$ is defined to be (orbitally) stable in $H^{\frac{\alpha}{2}}$ if

(i) there is a Banach space $Y \subset H^{\frac{\alpha}{2}}$ such that for all $u_{0} \in Y$, there is a unique solution $u$ of (1.1) in $C(\mathbb{R} ; Y) \subset C\left(\mathbb{R} ; H^{\frac{\alpha}{2}}\right)$ with $u(x, 0)=u_{0}$; and

(ii) for every $\epsilon>0$, there exists a $\delta>0$ such that for all $u_{0} \in U_{\delta} \cap Y$, the solution $u$ of (1.1) with $u(x, 0)=u_{0}$ satisfies $u(t) \in U_{\epsilon}$ for all $t>0$.

In the case $u \in C\left(\left(-T^{*}, T^{*}\right) ; Y\right) \subset C\left(\left(-T^{*}, T^{*}\right) ; H^{\frac{\alpha}{2}}\right)$, where $T^{*}$ is the maximal time of existence of $u$, the property of stability is called conditional.

Our first theorem of orbital stability for (1.1) with a "lower" dispersion (more exactly, of conditional type for $\alpha \in\left(\frac{1}{2}, 1\right)$, see Remark 2.1 below) is the following ([1], [14], [35]).

Theorem 1.1. [nonlinear stability of the ground state] Let $\frac{1}{2}<\alpha<2$ and $0<$ $p<p_{\max }(\alpha)$. Then the ground state solution $Q_{c}$ for equation (1.6) is $H^{\frac{\alpha}{2}}(\mathbb{R})$-stable by the flow of equation (1.1) for $p<2 \alpha$. 
Now, with regard to the stability (linear instability) properties of the solitary waves for (1.6) in the case $\alpha \in\left(\frac{1}{3}, \frac{1}{2}\right]$ and $p=1$ in (1.1), by considering the new variable

$$
w(x, t)=u(x+c t, t)-\varphi_{c}(x)
$$

into the fKdV equation and using equation (1.6) satisfied by $\varphi_{c}$, one finds that $w$ satisfies the nonlinear equation

$$
\left(\partial_{t}-c \partial_{x}\right) w+\partial_{x}\left(\varphi_{c} w-D^{\alpha} w+O\left(\|w\|^{2}\right)\right)=0 .
$$

As a leading approximation for small perturbation, we replace (1.30) by its linearization around $\varphi_{c}$, and hence obtain the linear equation

$$
\left(\partial_{t}-c \partial_{x}\right) w+\partial_{x}\left(\varphi_{c} w-D^{\alpha} w\right)=0 .
$$

Since $\varphi_{c}$ depends on $x$ and but not $t$, the equation (1.31) admits treatment by separation of variables, which leads naturally to a spectral problem. Seeking particular solutions of (1.31) of the form $w(x, t)=e^{\lambda t} u(x)$ (so-called growing mode solution), where $\lambda \in \mathbb{C}, u$ satisfies the linear problem

$$
\left(\lambda-c \partial_{x}\right) u+\partial_{x}\left(\varphi_{c} u-D^{\alpha} u\right)=0 .
$$

We can say from (1.32) that the complex growth rate $\lambda$ appears as (spectral) parameter for the extended eigenvalue problem

$$
\partial_{x} \mathcal{L}_{c} u=\lambda u,
$$

with $\mathcal{L}_{c}$ defined in (1.23) with $p=1$. If equation (1.33) have a nonzero solution $u \in$ $D\left(\mathcal{L}_{c}\right)=H^{\alpha}(\mathbb{R})$ then an bootstrapping argument shows that $u \in H^{s}(\mathbb{R})$ for all $s \geqq 1$, so that (1.33) is satisfied in classical sense. A necessary condition for the "stability" of $\varphi_{c}$ is that there are not points $\lambda$ with $\operatorname{Re}(\lambda)>0$ (which would imply the existence of a solution $u=u(x)$ of (1.33) that grows exponentially in time). If we denote by $\sigma$ the "spectrum" of $\partial_{x} \mathcal{L}_{c}$ (namely, $\lambda \in \sigma$ if there is a $u \neq 0$ satisfying (1.33)), the later discussion suggests the utility of the following definition:

Definition 1.3. (linear stability and instability) A solitary wave solution $\varphi_{c}$ of the fKdV equation (1.1) is said to be linearly stable if $\sigma \subset i \mathbb{R}$. Otherwise (i.e., if $\sigma$ contains point with $\operatorname{Re}(\lambda)>0$ ) $\varphi_{c}$ is linearly unstable.

We recall that as (1.31) is a real Hamiltonian equation, it forces certain elementary symmetries on the spectrum of $\sigma$, more exactly, $\sigma$ will be symmetric with respect to reflection in the real and imaginary axes. Therefore, it implies that exponentially growing perturbation are always paired with exponentially decaying ones. It is the reason by which was only required in Definition 1.3 that the spectral parameter $\lambda$ satisfies that $\operatorname{Re}(\lambda)>0$.

An similar spectral problem to (1.33) for traveling wave solutions (solitary or periodic) has been the focus of many research studies in the last years, see Grillakis\&Shatah\&Strauss [20], Lopes [38], Lin [34], Kapitula\&Stefanov [25], among others.

Our linearized instability result for the fKdV equation (1.1) is the following: 
Theorem 1.2. [Linear instability criterium for fKdV equations] Let $c \rightarrow \varphi_{c} \in$ $H^{\alpha+1}(\mathbb{R})$ be a smooth curve of positive solitary wave solutions to equation (1.6) with $\alpha \in\left(\frac{1}{3}, \frac{1}{2}\right), p=1$. The wave-speed $c$ can be considered over some nonempty interval $I$, $I \subset(0,+\infty)$. We assume that the self-adjoint operator $\mathcal{L}_{c}=D^{\alpha}+c-\varphi_{c}$ with domain $D\left(\mathcal{L}_{c}\right)=H^{\alpha}(\mathbb{R})$ satisfies

$$
\operatorname{Ker}\left(\mathcal{L}_{c}\right)=\left[\frac{d}{d x} \varphi_{c}\right]
$$

Denote by $n\left(\mathcal{L}_{c}\right)$ the number (counting multiplicity) of negative eigenvalues of the operator $\mathcal{L}_{c}$. Then there is a purely growing mode $e^{\lambda t} u(x)$ with $\lambda>0, u \in H^{s}(\mathbb{R})-\{0\}, s \geqq 0$, to the linearized equation (1.31) if one of the following two conditions is true:

(i) $n\left(\mathcal{L}_{c}\right)$ is even and $\frac{d}{d c}\left\langle\varphi_{c}, \varphi_{c}\right\rangle>0$.

(ii) $n\left(\mathcal{L}_{c}\right)$ is odd and $\frac{d}{d c}\left\langle\varphi_{c}, \varphi_{c}\right\rangle<0$.

The proof of the instability criterium established in Theorem 1.2 is based in the compactness of some specific commutators associated to the family $\mathcal{A}^{\lambda}$ defined in (2.9) below, and the analytic and asymptotic perturbation theory for linear operators. These approach can be also applied to the general model (1.2) with a linear operator $\mathcal{M}$ of "lower dispersion" under some specific conditions about the symbol $\beta$. Also, our approach can be extend to the case of periodic traveling waves solutions associated to the model (1.2) (a work in progress).

Remark 1.1. 1) The conditions $(i)-(i i)$ in Theorem 1.2 are similar to that obtained in Lin [34], case $\alpha \geqq 1$, and in Kapitula\&Stefanov [25]-Corollary 16.

2) Our approach provides the existence of a nonzero solution $u \in D\left(\mathcal{L}_{c}\right)=H^{\alpha}(\mathbb{R})$ satisfying the eigenvalue problem (1.33), via a different approach than that given in KapitulaßStefanov [25] and Pelinovsky [45].

3) If there is $\psi \in D\left(\mathcal{L}_{c}\right)$ such that $\mathcal{L}_{c} \psi=\varphi_{c}$, the conditions $(i)-(i i)$ in Theorem 1.2 can be changed by

(i) $n\left(\mathcal{L}_{c}\right)$ is even and $\left\langle\psi, \varphi_{c}\right\rangle<0$.

(ii) $n\left(\mathcal{L}_{c}\right)$ is odd and $\left\langle\psi, \varphi_{c}\right\rangle>0$.

4) The former criterium 3) is very useful when we do not have in hands a smooth curve $c \rightarrow \varphi_{c}$ of solitary waves.

As consequence of Theorem 1.2 we obtain the following stability result for the ground state solutions of equation (11.6) (see [25]).

Corollary 1.1. [Linear instability of ground state for fKdV equations] For $\alpha \in$ $\left(\frac{1}{3}, \frac{1}{2}\right), p=1$ and $c>0$, the ground state profiles $Q_{c}$ for (1.6) are spectrally unstable.

Next, we consider $u(x, t)=\psi_{c}(x-c t)$ a solitary wave solution for the model (1.2). Then $\psi_{c}$ satisfies

$$
\mathcal{M} \psi_{c}+c \psi_{c}-f\left(\psi_{c}\right)=0
$$


and, similarly as in the case of model (1.1), we also have the linearized equation around $\psi_{c}$

$$
\left(\partial_{t}-c \partial_{x}\right) u+\partial_{x}\left(f^{\prime}\left(\psi_{c}\right) u-\mathcal{M} u\right)=0 .
$$

In order to obtain a growing mode solution of the form $e^{\lambda t} w(x), \operatorname{Re} \lambda>0$, function $w$ must satisfy

$$
\left(\lambda-c \partial_{x}\right) w+\partial_{x}\left(f^{\prime}\left(\psi_{c}\right) w-\mathcal{M} w\right)=0 .
$$

Then similarly as in the case of model (1.1) we obtain the following linearized instability result for the gfKdV equation (1.2), provided that the symbol $\beta$ defining the pseudodifferential operator $\mathcal{M}$ satisfies for $\alpha \in(0,1)$ the following condition:

$$
\text { if } \eta(\xi) \equiv \beta(\xi)-|\xi|^{\alpha} \quad \text { then } \quad \eta^{\prime} \in L^{2}(\mathbb{R}) \text {. }
$$

Theorem 1.3. [Linear instability criterium for gfKdV equation] Let $c \in I \subset$ $(0,+\infty) \rightarrow \psi_{c} \in H^{\alpha+1}, 0<\alpha<1$, be a smooth curve of positive solitary wave solutions to equation (1.35). We assume condition (1.38) and that the self-adjoint operator $\mathcal{N}_{c}=$ $\mathcal{M}+c-f^{\prime}\left(\psi_{c}\right)$ with domain $D\left(\mathcal{N}_{c}\right)=H^{\alpha}(\mathbb{R})$ satisfies

$$
\operatorname{Ker}\left(\mathcal{N}_{c}\right)=\left[\frac{d}{d x} \psi_{c}\right]
$$

Denote by $n\left(\mathcal{N}_{c}\right)$ the number (counting multiplicity) of negative eigenvalues of the operator $\mathcal{N}_{c}$. Then there is a purely growing mode $e^{\lambda t} w(x)$ with $\lambda>0, w \in H^{s}(\mathbb{R})-\{0\}, s \geqq 0$, to the linearized equation (1.36) if one of the following two conditions is true:

(i) $n\left(\mathcal{N}_{c}\right)$ is even and $\frac{d}{d c}\left\langle\psi_{c}, \psi_{c}\right\rangle>0$.

(ii) $n\left(\mathcal{N}_{c}\right)$ is odd and $\frac{d}{d c}\left\langle\psi_{c}, \psi_{c}\right\rangle<0$.

The proof of Theorem 1.3 follows the same lines of that for Theorem 1.2 , but because of the generality of the symbol associated with the operator $\mathcal{M}$ some points in the analysis need to be treated carefully.

The analysis established above for the fKdV equation (1.1) can be extend to the fBBM equation (1.5) for $\alpha \in\left(\frac{1}{3}, 1\right)$. In section 4 below, we show the following stability properties associated to the ground state solutions $\Phi_{c}$ satisfying

$$
D^{\alpha} \Phi_{c}+\left(1-\frac{1}{c}\right) \Phi_{c}-\frac{1}{c} \Phi_{c}^{2}=0, \quad c>1 .
$$

Theorem 1.4. [nonlinear stability of the ground state for the fBBM] Let $\frac{1}{3}<$ $\alpha<1$ and $c>1$. Then, the ground state solution $\Phi_{c}$ of $(1.40)$ is $H^{\frac{\alpha}{2}}(\mathbb{R})$-stable by the flow of equation (1.5) provided $\alpha \in\left[\frac{1}{2}, 1\right)$ and $c>1$, and for $\alpha \in\left(\frac{1}{3}, \frac{1}{2}\right)$ and $c>c_{0}$. Here $c_{0}$ is given by

$$
c_{0}=\frac{2+\sqrt{2(3 \alpha-1)}}{6 \alpha} .
$$


Our nonlinear stability results for the fBBM equation extends and complements those in Linares\&Pilod\&Saut [35], in the sense that we show stability of the orbit $\Omega_{\Phi_{c}}=\left\{\Phi_{c}(\cdot+y)\right.$ :

$y \in \mathbb{R}\}$ for $\alpha=\frac{1}{2}$ and $c>1$, and for $\frac{1}{3}<\alpha<\frac{1}{2}$ with the specific restriction on the wave velocity $c$. It also confirms the numerical simulations in Klein\&Saut [29] about the stability of the solitary waves in this regimen of $\alpha$ 's. Similarly as in the case of the fKdV equation, the statement of orbital stability in Theorem 1.4 is a conditional one (see Remark 4.1 below).

Theorem 1.5. [Linear instability of ground state for fBBM equations] For $\alpha \in$ $\left(\frac{1}{3}, \frac{1}{2}\right)$ and $c \in\left(1, c_{0}\right)$, the ground state profiles $\Phi_{c}$ for 1.40) are linearly unstable.

Our paper is organized as follows. In Section 2 we present the proof of the linear instability criterium in Theorem 1.2 for the fKdV model (1.1) and of the criterium for the general dispersive equation (1.2) in Theorem 1.3. In Section 3, we prove our "stability of the blow-up" for the critical fKdV equation (1.1) $\left(\alpha=\frac{1}{2}, p=1\right)$. In the final section 4 , we prove the results of nonlinear stability and linear instability for the fBBM equation (1.5)) (Theorems 1.4 and 1.5).

Notation. We will denote $|\cdot|_{p}$ the norm in the Lebesgue space $L^{p}(\mathbb{R}), 1 \leqq p \leqq \infty$ and $\|\cdot\|_{s}$ the norm in the Sobolev space $H^{s}(\mathbb{R}), s \in \mathbb{R}$. For $X, Y$ Banach spaces, $B(X ; Y)$ represents the set of bounded linear operators $F: X \rightarrow Y$. [A, $B]=A B-B A$ represents the commutator of the operators $A$ and $B . \rho(A)$ will represent the resolvent of the linear operator $A$.

\section{Nonlinear Stability AND Linear Instability fOR THE FKDV EQUATION}

This section is devoted to show Theorem 1.1 and Theorem 1.2 established in the introduction. The proof of the nonlinear stability of the ground state is a consequence immediate of Grillakis et.al [19] and Frank\&Lenzmann [16] results. For the linear instability result we extend the theory of Vock\&Hunziker in [48] about the stability of Schrödinger eigenvalue problems to the study of linear instability of solitary waves solutions for the fKdV type model in (1.1) with a "lower dispersion". In particular, we recover the linear instability results in [25] for the ground state solutions associated to the equation (1.6) with $p=1$ and $\alpha \in\left(\frac{1}{3}, \frac{1}{2}\right)$. Our analysis is also extended to the general lower-dispersion models (1.2).

2.1. Nonlinear stability of ground state for the fKdV equation. In the following we show Theorem 1.1, we recall that in the literature it result of stability has been showed by difference methods ([1], [14, [35]). Here, for completeness of the exposition, we show this in a unified way for $\alpha \in\left(\frac{1}{2}, 2\right)$.

Proof. [Proof of Theorem 1.1] Let $Q$ be the ground state solution for (1.10), namely, $Q=Q(|x|)>0$, satisfies

$$
D^{\alpha} Q+Q-Q^{2}=0
$$


and a minimum for the functional $J^{\alpha, 1}$ in (1.11). Thus we obtain that the self-adjoint operator,

$$
\mathcal{L}_{1}=D^{\alpha}+1-2 Q
$$

satisfies the so-called nondegeneracy property, namely, $\operatorname{Ker}\left(\mathcal{L}_{1}\right)=\left[\frac{d}{d x} Q\right]$. Moreover, since $\left\langle\mathcal{L}_{1} Q, Q\right\rangle \leqq 0$ and for $\eta \in C_{0}^{\infty}(\mathbb{R})$

$$
\left\langle\mathcal{L}_{1} \eta, \eta\right\rangle \geqq 0, \quad \text { for all } \eta \perp Q^{2},
$$

we have $n\left(\mathcal{L}_{1}\right)=1$ (see [16]). Now, for $R \equiv \alpha Q+x Q^{\prime} \in L^{2}(\mathbb{R})$ (see (1.14)) follows $\mathcal{L}_{1} R=-\alpha Q$ (at least in the distributional sense). Thus a bootstrapping argument shows that $R \in H^{\alpha+1}(\mathbb{R})$ and so $R \in D\left(\mathcal{L}_{1}\right)=H^{\alpha}(\mathbb{R})$.

Next, for any real number $\theta \neq 0$, define the dilation operator $T_{\theta}$ by $\left(T_{\theta} f\right)(x)=f(\theta x)$. Then, via the elementary scaling $Q_{c}(x)=2 c Q\left(c^{1 / \alpha} x\right)$ and the relation $D^{\alpha}\left(T_{\theta} f\right)(x)=$ $\theta^{\alpha} D^{\alpha} f(\theta x)$, we can show that for $\theta=c^{1 / \alpha}$ we obtain that $Q_{c}$ satisfies

$$
D^{\alpha} Q_{c}+c Q_{c}-\frac{1}{2} Q_{c}^{2}=0
$$

and so, we obtain its linearized operator

$$
\mathcal{L}_{c}=D^{\alpha}+c-Q_{c}
$$

Now, the relation $\mathcal{L}_{c}=c T_{\theta} \mathcal{L}_{1} T_{\theta}^{-1}$ implies with $\theta=c^{1 / \alpha}$ that $\operatorname{spec}\left(\mathcal{L}_{c}\right)=\{c r: r \in$ $\left.\operatorname{spec}\left(\mathcal{L}_{1}\right)\right\}$ and therefore $\mathcal{L}_{c}$ and $\mathcal{L}_{1}$ have the "same structure". Thus, $\psi$ is an eigenfunction of $\mathcal{L}_{1}$ with eigenvalue $\lambda$ if and only if $T_{\theta} \psi$ is an eigenfunction of $\mathcal{L}_{c}$ with eigenvalue $c \lambda$. Then, we conclude immediately that $n\left(\mathcal{L}_{c}\right)=1$ and $\operatorname{Ker}\left(\mathcal{L}_{c}\right)=\left[\frac{d}{d x} Q_{c}\right]$. Thus, since $R_{c}=$ $\alpha Q_{c}+x Q_{c}^{\prime} \in D\left(\mathcal{L}_{c}\right)$ with $\mathcal{L}_{c} R_{c}=-\alpha c Q_{c}\left(\right.$ where we are used $\left.D^{\alpha}\left(x Q^{\prime}\right)=\alpha D^{\alpha} Q+x D^{\alpha} Q^{\prime}\right)$ we obtain

$$
\left\langle\mathcal{L}_{c}^{-1} Q_{c}, Q_{c}\right\rangle=-\frac{1}{\alpha c}\left\langle R_{c}, Q_{c}\right\rangle=\left\|Q_{c}\right\|^{2}\left[\frac{1}{2 \alpha c}-\frac{1}{c}\right]<0,
$$

where we use integration by parts $\left(x Q_{c}^{2}(x) \rightarrow 0\right.$ as $\left.|x| \rightarrow+\infty\right)$ and $\alpha>\frac{1}{2}$. Hence, from regularity properties of the curve $c \rightarrow Q_{c}$ (see proof of Corollary 1.1 below) and from the Lyapunov property of the energy $E$ in (1.21) we finish the proof.

Remark 2.1. The statement in Theorem 1.1 deserves to be clarified at least in some points with regard to the Cauchy problem.

(1) For $2>\alpha>1$ the solutions of the Cauchy problem are global in $H^{\frac{\alpha}{2}}(\mathbb{R})$ and so the stability result is not conditional (see Definition 1.2). Indeed, by using a similar strategy to that in the proof of Theorem 1 in Kenig\&MartelERobbiano [27] we obtain local well-posedness of the model in (1.1) for every initial data $u_{0} \in H^{\frac{\alpha}{2}}(\mathbb{R})$ for $p<2 \alpha$ (in [27] was studied the case of the critical-nonlinearity $\left.|u|^{2 \alpha} u_{x}\right)$. Moreover, the conservation of the energy $E$ in (1.16) and the charge 
$F(u)=\int_{\mathbb{R}} u^{2} d x$ by the flow of (1.1), together with an application of the GagliardoNirenberg type inequality (see (1.12))

$$
\int_{\mathbb{R}}|u|^{p+2} \leqq C_{\alpha, p}\left(\int_{\mathbb{R}}\left|D^{\frac{\alpha}{2}} u\right|^{2}\right)^{\frac{p}{2 \alpha}}\left(\int_{\mathbb{R}}|u|^{2}\right)^{\frac{p}{2 \alpha}(\alpha-1)+1},
$$

it gives us exactly for $p<2 \alpha$ the "a priori" estimative,

$$
\begin{aligned}
\left\|D^{\frac{\alpha}{2}} u\right\|^{2} & \leqq E\left(u_{0}\right)+C_{\alpha, p}\left\|D^{\frac{\alpha}{2}} u\right\|^{\frac{p}{\alpha}}\|u\|^{\beta} \\
& \leqq C\left(\left\|u_{0}\right\|_{\frac{\alpha}{2}}\right)+\frac{D_{\alpha, p}}{r}\left\|u_{0}\right\|^{p r+2}+\frac{p}{2 \alpha}\left\|D^{\frac{\alpha}{2}} u\right\|^{2}
\end{aligned}
$$

with $\beta=\frac{p}{\alpha}(\alpha-1)+2$ and $r=\frac{2 \alpha}{2 \alpha-p}$. Thus, global-posedness of the initial valued problem for (1.1) follows in the energy space $H^{\frac{\alpha}{2}}(\mathbb{R})$.

(2) In Herr\&Ionescu\&Kenig\&Koch [21] was showed that for $\alpha \in(1,2)$ the solutions of the Cauchy problem for (1.1) with $p=1$ are global well-posed in $L^{2}(\mathbb{R})$.

(3) The case $\alpha=1$ ( $p=1$, that is, the Benjamin-Ono equation) was showed to be globally well-posed in $H^{s}(\mathbb{R})$ for $s \geqq 0$ by Ionescu\& Kenig [23].

(4) The case $\alpha \in\left(\frac{1}{2}, 1\right)$ is more delicate with regard to the local and global wellposedness problem. In Saut [46] was proved that (1.1) admit global weak solutions (without uniqueness) in the space $L^{\infty}\left(\mathbb{R} ; H^{\frac{\alpha}{2}}(\mathbb{R})\right)$ and global weak solutions in $L^{\infty}\left(\mathbb{R} ; L^{2}(\mathbb{R})\right) \cap L_{\text {loc }}^{2}\left(\mathbb{R} ; H_{\text {loc }}^{\frac{\alpha}{2}}(\mathbb{R})\right)$ in Ginibre\& Velo [17]-[18].

(5) The best known result of local well-posedness for (1.1) has been established by LinaresEPilodESaut [35] in $H^{s}(\mathbb{R})$, for $s>s_{\alpha} \equiv \frac{3}{2}-\frac{3 \alpha}{8}>\frac{\alpha}{2}$, for $\alpha \in(0,1)$. It which does not allow to globalize the solution using conservation laws.

(6) The problem to prove local well-posedness in $H^{\frac{\alpha}{2}}(\mathbb{R})$ in the case $\alpha \in\left[\frac{1}{2}, 1\right)$, which would imply global well-posedness by using the conserved quantities $E$ and $F$, is still open.

(7) Therefore, the statement of stability in Theorem 1.1 for $\alpha \in\left(\frac{1}{2}, 1\right)$ is one of conditional type by the Definition 1.2, where we have used $Y=H^{s}(\mathbb{R}), s>s_{\alpha}>\frac{1}{2}$, and so for all $\epsilon>0$ there is a $\delta>0$ such that if $u_{0} \in H^{s}(\mathbb{R}) \cap U_{\delta}$, then $u(t) \in U_{\epsilon}$, for all $t \in\left(-T_{s}, T_{s}\right)$, where $T_{s}$ is the maximal time of existence of $u$ satisfying $u(0)=u_{0}$.

2.2. linear instability criterium for the $\mathbf{f K d V}$ equation. In order to illustrate the strategy for obtaining a growing mode solution of (1.31) with the form $w(x, t)=e^{\lambda t} u(x)$ and $\operatorname{Re} \lambda>0$, we can see the eigenvalue problem (1.32) for $\lambda$ and $u$ rewrite in the form

$$
c u+\frac{c \partial_{x}}{\lambda-c \partial_{x}}\left(\varphi_{c} u-D^{\alpha} u\right)=0 .
$$

Here the expression $\frac{\partial_{x}}{\lambda-c \partial_{x}}$ is a notation for the well-defined linear operator $\partial_{x}\left(\lambda-c \partial_{x}\right)^{-1}$ and $\varphi_{c}$ is any positive solitary wave solutions for (1.6). Thus, if we consider the following 
family of closed linear operators $\mathcal{A}^{\lambda}: H^{\alpha}(\mathbb{R}) \longrightarrow L^{2}(\mathbb{R})$, with $\operatorname{Re} \lambda>0$,

$$
\mathcal{A}^{\lambda} v \equiv c v+\frac{c \partial_{x}}{\lambda-c \partial_{x}}\left(\varphi_{c} v-D^{\alpha} v\right)
$$

it follows immediately that the solution of the eigenvalue problem (2.8) is reduced to find $\lambda \in \mathbb{C}$ with $\operatorname{Re} \lambda>0$ such that the operator $\mathcal{A}^{\lambda}$ possesses a nontrivial kernel. Now, we note that from the analyticity of the resolvent associated to the operator $\partial_{x}, \lambda \in \mathcal{S} \rightarrow$ $\left(\lambda-c \partial_{x}\right)^{-1}$, for $\mathcal{S}=\{z \in \mathbb{C}: \operatorname{Re} z>0\}$, we obtain that the mapping $\lambda \in \mathcal{S} \rightarrow \mathcal{A}^{\lambda}$ represents an analytical family of operators of type- $A$ (see Kato [26]), namely,

1) $D\left(\mathcal{A}^{\lambda}\right)=H^{\alpha}(\mathbb{R})$ for all $\lambda \in \mathcal{S}$,

2) for $u \in H^{\alpha}(\mathbb{R}), \lambda \in \mathcal{S} \rightarrow \mathcal{A}^{\lambda} u$ is analytic in the topology of $L^{2}(\mathbb{R})$.

Therefore, from classical analytic perturbation theory, all discrete eigenvalues of $\mathcal{A}^{\lambda}$ $(\operatorname{Re}(\lambda)>0)$ will be stable : for $\eta$ in the discrete spectrum of $\mathcal{A}^{\lambda}$, there is $\delta>0$ such that for $\lambda_{0} \in B(\lambda ; \delta), \mathcal{A}^{\lambda_{0}}$ has $\eta_{i}\left(\lambda_{0}\right)$ eigenvalues close to $\eta$ with total algebraic multiplicity equal to that of $\eta$.

In our approach, we will find a growing mode solution for $\lambda>0$. Indeed, since we have that

$$
\mathcal{A}^{\lambda} \longrightarrow \mathcal{L}_{c} \equiv D^{\alpha}+c-\varphi_{c} \quad \text { as } \lambda \rightarrow 0^{+},
$$

strongly in $L^{2}(\mathbb{R})$ (see Proposition 2.1 below), we will use asymptotic perturbation arguments as Vock\&Hunziker in [48] and Lin in [34] (see also Hislop\&Sigal in [22]) for obtaining our criterium established in Theorem 1.2. In our analysis, it will be decisive to count the number of eigenvalues of $\mathcal{A}^{\lambda}$ (for $\lambda$ small) in the left-half plane (for $\lambda$ large, there is not growing modes, see Lemma 2.3 below), and so we will need to know how the zero eigenvalue of $\mathcal{L}_{c}$ will be perturbed. To this end, we obtain a moving kernel formula (see Lemma 2.6 below) which will decide whether zero jumps to the left or to the right. Thus we get the conditions $(i)-(i i)$ in Theorem 1.2 .

As the structure of the proof of Theorem 1.2 follows some ideas used by Lin in [34] for the case $\alpha \geqq 1$, we will only indicate the new basic differences due to the structure of the operator $\mathcal{A}^{\bar{\lambda}}$ defined in $(2.9)$ for $\alpha \in(0,1)$.

2.2.1. Stability of the discrete spectrum of $\mathcal{L}_{c}$, with $\alpha \in(0,1)$. In this subsection we study the behavior of the family $\mathcal{A}^{\lambda}$ by depending of $\lambda$. In particular, we show that every discrete eigenvalue of the limiting operator $\mathcal{L}_{c}=D^{\alpha}+c-\varphi_{c}$ is stable with respect to the family $\mathcal{A}^{\lambda}$ for small positive $\lambda$. Our first result is about the strong convergence of $\mathcal{A}^{\lambda}$.

Proposition 2.1. For $\lambda>0$, the operator $\mathcal{A}^{\lambda}$ converges to $\mathcal{L}_{c}$ strongly in $L^{2}(\mathbb{R})$ when $\lambda \rightarrow 0^{+}$.

Proof. For $\lambda>0$ and $v \in D\left(\mathcal{A}^{\lambda}\right)=D\left(\mathcal{L}_{c}\right)$ we have the relation

$$
\left(\mathcal{A}^{\lambda}-\mathcal{L}_{c}\right) v=\frac{\lambda}{\lambda-c \partial_{x}}\left(\varphi_{c}-D^{\alpha}\right) v
$$


Thus, by Plancherel and the dominated convergence theorem follows

$$
\left\|\left(\mathcal{A}^{\lambda}-\mathcal{L}_{c}\right) v\right\|^{2}=\int_{\mathbb{R}} \frac{\lambda^{2}}{\lambda^{2}+c^{2} \xi^{2}}\left|\widehat{\varphi}_{c}(\xi)-\widehat{D^{\alpha}}(\xi)\right|^{2} d \xi \rightarrow 0
$$

when $\lambda \rightarrow 0^{+}$.

Next, we localized the essential spectrum of $\mathcal{A}^{\lambda}, \sigma_{\text {ess }}\left(\mathcal{A}^{\lambda}\right)$. We will see that it set is situated in the right-hand side of the complex-plane and away from the imaginary axis. We star with the following two basic definition related to the $\sigma_{e s s}\left(\mathcal{A}^{\lambda}\right)$ (see Hislop\&Sigal [22]).

Definition 2.1. [Zhislin spectrum $Z\left(\mathcal{A}^{\lambda}\right)$ ] $A$ Zhislin sequence for $\mathcal{A}^{\lambda}$ and $z \in \mathbb{R}$ is a sequence

$$
\left\{u_{n}\right\} \subset H^{\alpha}(\mathbb{R}), \quad\left\|u_{n}\right\|=1, \quad \text { supp } u_{n} \subset\{x:|x| \geqq n\}
$$

and $\left\|\left(\mathcal{A}^{\lambda}-z\right) u_{n}\right\| \rightarrow 0$ as $n \rightarrow+\infty$.

The set of all $z$ such that a Zhislin sequence exists for $\mathcal{A}^{\lambda}$ and $z$ is denoted by $Z\left(\mathcal{A}^{\lambda}\right)$.

Remark 2.2. Every Zhislin sequence $\left\{u_{n}\right\}$ necessarily converges weakly to zero in $L^{2}(\mathbb{R})$.

Definition 2.2. [Weyl spectrum $W\left(\mathcal{A}^{\lambda}\right)$ ] $A$ Weyl sequence for $\mathcal{A}^{\lambda}$ and $z \in \mathbb{R}$ is a sequence

$$
\left\{u_{n}\right\} \subset H^{\alpha}(\mathbb{R}), \quad\left\|u_{n}\right\|=1, \quad u_{n} \rightarrow 0 \text { weakly in } L^{2}(\mathbb{R}),
$$

and $\left\|\left(\mathcal{A}^{\lambda}-z\right) u_{n}\right\| \rightarrow 0$ as $n \rightarrow+\infty$.

The set of all $z$ such that a Weyl sequence exists for $\mathcal{A}^{\lambda}$ and $z$ is denoted by $W\left(\mathcal{A}^{\lambda}\right)$.

From the last two definitions we have the following result (see [22]).

Proposition 2.2. $Z\left(\mathcal{A}^{\lambda}\right) \subset W\left(\mathcal{A}^{\lambda}\right), W\left(\mathcal{A}^{\lambda}\right) \subset \sigma_{\text {ess }}\left(\mathcal{A}^{\lambda}\right)$ and $\partial\left(\sigma_{\text {ess }}\left(\mathcal{A}^{\lambda}\right)\right) \subset W\left(\mathcal{A}^{\lambda}\right)$.

Our main result about the $\sigma_{\text {ess }}\left(\mathcal{A}^{\lambda}\right)$ is the following one.

Proposition 2.3. For any $\lambda>0$, we have

$$
\sigma_{\text {ess }}\left(\mathcal{A}^{\lambda}\right) \subset\left\{z: \operatorname{Re} z \geqq \frac{1}{2} c\right\}
$$

The idea of the proof of Proposition 2.3 will be to see $W\left(\mathcal{A}^{\lambda}\right)=Z\left(\mathcal{A}^{\lambda}\right)$ and it will be based on the following two lemmas.

Lemma 2.1. For any $\lambda>0$, we have

$$
Z\left(\mathcal{A}^{\lambda}\right) \subset\left\{z: R e z \geqq \frac{1}{2} c\right\} .
$$


Proof. Let $z \in Z\left(\mathcal{A}^{\lambda}\right)$ and suppose $\operatorname{Rez}<\frac{1}{2} c$. It is immediate from Fourier transform that for any $u \in H^{\alpha}(\mathbb{R})$ we have

$$
I_{0}(u) \equiv R e\left\langle-\frac{c \partial_{x}}{\lambda-c \partial_{x}} D^{\alpha} u, u\right\rangle \geqq 0 .
$$

Then, for any sequence $\left\{u_{n}\right\} \subset H^{\alpha}(\mathbb{R}),\left\|u_{n}\right\|=1$, and satisfying supp $u_{n} \subset\{x:|x| \geqq n\}$, we have from the following trivial estimative for any $c$ and $\lambda$

$$
\left\|\frac{c \partial_{x}}{\lambda+c \partial_{x}} u_{n}\right\| \leqq\left\|u_{n}\right\|=1
$$

that for $n$ large,

$$
\begin{aligned}
\operatorname{Re}\left\langle\left(\mathcal{A}^{\lambda}-z\right) u_{n}, u_{n}\right\rangle & \geqq I_{0}\left(u_{n}\right)+c-\operatorname{Re} z+\operatorname{Re}\left\langle\frac{c \partial_{x}}{\lambda-c \partial_{x}}\left(u_{n} \varphi_{c}\right), u_{n}\right\rangle \\
& \geqq c-\operatorname{Re} z-\operatorname{Re}\left\langle u_{n} \varphi_{c}, \frac{c \partial_{x}}{\lambda+c \partial_{x}} u_{n}\right\rangle \\
& \geqq c-\operatorname{Re} z-\sup _{|x| \geqq n}\left|\varphi_{c}(x)\right| \geqq c-\frac{1}{2} c-\frac{1}{4} c=\frac{1}{4} c .
\end{aligned}
$$

Then, since $\left|\operatorname{Re}\left\langle\left(\mathcal{A}^{\lambda}-z\right) u_{n}, u_{n}\right\rangle\right| \leqq\left\|\left(\mathcal{A}^{\lambda}-z\right) u_{n}\right\|$, for all $n$, and $\left(\mathcal{A}^{\lambda}-z\right) u_{n} \rightarrow 0$ as $n \rightarrow+\infty$, we obtain a contradiction.

The next lemma extended Lemma 2.3 in [34] to the case $\alpha \in(0,1)$.

Lemma 2.2. Given $\lambda>0$. Let $\zeta \in C_{0}^{\infty}(\mathbb{R})$ be a cut-off function such that $\left.\zeta\right|_{\left\{|x| \leqq R_{0}\right\}}=1$, for some $R_{0}$. Define $\zeta_{d}(x)=\zeta(x / d), d>0$. Then, for each $d$, the operator $\zeta_{d}\left(\mathcal{A}^{\lambda}-z\right)^{-1}$ is compact for some $z \in \rho\left(\mathcal{A}^{\lambda}\right)$, and there exists $C(d) \rightarrow 0$ as $d \rightarrow \infty$ such that for any $u \in C_{0}^{\infty}(\mathbb{R})$,

$$
\left\|\left[\mathcal{A}^{\lambda}, \zeta_{d}\right] u\right\| \leqq C(d)\left(\left\|\mathcal{A}^{\lambda} u\right\|+\|u\|\right) .
$$

Proof. Initially we prove that for $k>0$ sufficiently large, $-k \in \rho\left(\mathcal{A}^{\lambda}\right)$. Indeed, for $\lambda>0$ we write $\mathcal{A}^{\lambda}=D^{\alpha}+c+\mathcal{K}^{\lambda}$, where

$$
\mathcal{K}^{\lambda}=\frac{c \partial_{x}}{\lambda-c \partial_{x}} \varphi_{c}-\frac{\lambda}{\lambda-c \partial_{x}} D^{\alpha}: L^{2}(\mathbb{R}) \rightarrow L^{2}(\mathbb{R})
$$

is a bounded operator, because of the symbol of $\partial_{x}\left(\lambda-c \partial_{x}\right)^{-1}$ and $\left(\lambda-c \partial_{x}\right)^{-1} D^{\alpha}$ are bounded (here we use that $\alpha<1$ ). Thus, since $A=D^{\alpha}+c$ is a nonnegative self-adjoint operator and $\mathcal{K}^{\lambda}$ is a $A$-bounded operator with relative $A$-bound equal to zero, we have that $-k \in \rho(A)$ for all $k>0$ and

$$
\left\|\mathcal{K}^{\lambda}(A+k)^{-1} u\right\| \leqq C_{\lambda, c}\left\|(A+k)^{-1} u\right\| \leqq C_{\lambda, c} \frac{1}{|k|}\|u\| .
$$

Therefore, the relation $\mathcal{A}^{\lambda}+k=\left[1+\mathcal{K}^{\lambda}(A+k)^{-1}\right](A+k)$ implies that for $k$ large, $z=-k \in \rho\left(\mathcal{A}^{\lambda}\right)$. Now, the compactness of $\zeta_{d}\left(\mathcal{A}^{\lambda}-z\right)^{-1}$ follows from the relation

$$
\left\|D^{\alpha}\left(\mathcal{A}^{\lambda}+k\right)^{-1} f_{n}\right\| \leqq\left\|\left[1+\mathcal{K}^{\lambda}(A+k)^{-1}\right]^{-1} f_{n}\right\| \leqq M\left\|f_{n}\right\|
$$


for all $L^{2}(\mathbb{R})$-bounded sequence $\left\{f_{n}\right\}$, the local compactness of $H^{\alpha}(\mathbb{R}) \hookrightarrow L^{2}(\mathbb{R})$ and a Cantor diagonalization argument which imply that $\left(\mathcal{A}^{\lambda}+k\right)^{-1} f_{n} \rightarrow f$ in $L_{l o c}^{2}(\mathbb{R})$ and so the sequence $\zeta_{d}\left(\mathcal{A}^{\lambda}-z\right)^{-1} f_{n}$ is convergent.

To show the commutator estimative in (2.12), we note initially that the graph norm of $\mathcal{A}^{\lambda}$ appearing at the right-side hand of $(2.12)$ is equivalent to the $\|\cdot\|_{H^{\alpha}}$-norm (it follows immediately from the relations $\mathcal{A}^{\lambda}=D^{\alpha}+c+\mathcal{K}^{\lambda}$ and $(A+k)=\left[1+\mathcal{K}^{\lambda}(A+k)^{-1}\right]^{-1}\left(\mathcal{A}^{\lambda}+\right.$ $k)$ ). Now, it is not difficult to see that $\mathcal{E}^{\lambda} \equiv \frac{\lambda}{\lambda-c \partial_{x}} \in B\left(L^{2}(\mathbb{R})\right)$ and we have the equality

$$
\left[\mathcal{A}^{\lambda}, \zeta_{d}\right]=\left(1-\mathcal{E}^{\lambda}\right)\left[D^{\alpha}, \zeta_{d}\right]+\left[\mathcal{E}^{\lambda}, \zeta_{d}\right]\left(\varphi_{c}-D^{\alpha}\right)
$$

Next, we estimative every term at the right-hand side of (2.14). First, from the relation

$$
\begin{aligned}
{\left[\mathcal{E}^{\lambda}, \zeta_{d}\right]\left(\varphi_{c}-D^{\alpha}\right) } & =\frac{1}{\lambda} \mathcal{E}^{\lambda}\left[c \partial_{x}, \zeta_{d}\right] \frac{1}{\lambda-c \partial_{x}}\left(\varphi_{c}-D^{\alpha}\right) \\
& =\frac{c}{\lambda d} \varepsilon^{\lambda}\left(\zeta^{\prime}(x / d) \mathcal{E}^{\lambda}\left(\varphi_{c}-D^{\alpha}\right)\right)
\end{aligned}
$$

we obtain

$$
\begin{aligned}
\left\|\left[\mathcal{E}^{\lambda}, \zeta_{d}\right]\left(\varphi_{c}-D^{\alpha}\right) u\right\| & \leqq \frac{c}{\lambda d}\left\|\zeta^{\prime}\right\|_{\infty}\left\|\varphi_{c} u-D^{\alpha} u\right\| \\
& \leqq \frac{C_{0}}{\lambda d}\left(\|u\|+\left\|D^{\alpha} u\right\|\right) .
\end{aligned}
$$

Now, since $1-\mathcal{E}^{\lambda} \in B\left(L^{2}(\mathbb{R})\right)$, we obtain from Theorem 3.3 in Murray [44] the estimative for $\alpha \in(0,1)$

$$
\left\|\left(1-\mathcal{E}^{\lambda}\right)\left[D^{\alpha}, \zeta_{d}\right] u\right\| \leqq\left\|\left[D^{\alpha}, \zeta_{d}\right] u\right\| \leqq K(\alpha)\left\|D^{\alpha} \zeta_{d}\right\|_{*}\|u\| \leqq \frac{C_{1}}{d^{\alpha}}\left\|D^{\alpha} \zeta\right\|_{\infty}\|u\|,
$$

where $\|\cdot\|_{*}$ is the BMO norm and we are using the identity $\left(D^{\alpha} \zeta_{d}\right)(x)=\frac{1}{d^{\alpha}}\left(D^{\alpha} \zeta\right)(x / d)$ and the classical embedding $L^{\infty}(\mathbb{R}) \hookrightarrow B M O\left(\|f\|_{*} \leqq 2\|f\|_{\infty}\right)$. Therefore, from (2.14)-(2.15)(2.16) we obtain the right-hand side in (2.12). It finishes the proof of the Lemma.

Proof. [Proposition 2.3]. By using Theorem 10.12 in 22] and Lemma 2.2 above, we have for any $\lambda>0$ that $W\left(\mathcal{A}^{\lambda}\right)=Z\left(\mathcal{A}^{\lambda}\right)$. Therefore, Lemma 2.1 and Proposition 2.2 imply Proposition 2.3. Indeed, suppose that that $z \in \sigma_{\text {ess }}\left(\mathcal{A}^{\lambda}\right)$ and $\operatorname{Re} z<\frac{1}{2} c$. Then $z \in \mathbb{C}-W\left(\mathcal{A}^{\lambda}\right) \subset \mathbb{C}-\partial\left(\sigma_{\text {ess }}\left(\mathcal{A}^{\lambda}\right)\right)$. Therefore, $z \in \operatorname{Int}\left(\sigma_{\text {ess }}\left(\mathcal{A}^{\lambda}\right)\right)$. So, if we consider $C_{z}$ being the maximal non-empty open connected component of $\operatorname{Int}\left(\sigma_{\text {ess }}\left(\mathcal{A}^{\lambda}\right)\right)$ containing the point $z$, we see that $\partial C_{z} \cap\left\{z: \operatorname{Re} z<\frac{1}{2} c\right\} \neq \emptyset$. Therefore, since $\partial C_{z} \subset \partial\left(\sigma_{\text {ess }}\left(\mathcal{A}^{\lambda}\right)\right)$ we obtain $\partial\left(\sigma_{\text {ess }}\left(\mathcal{A}^{\lambda}\right)\right) \cap\left\{z: \operatorname{Re} z<\frac{1}{2} c\right\} \neq \emptyset$ and so $W\left(\mathcal{A}^{\lambda}\right) \cap\left\{z: \operatorname{Re} z<\frac{1}{2} c\right\} \neq \emptyset$, it which is a contradiction. This finishes the proof. 
Next, we study the behavior of $\mathcal{A}^{\lambda}$ near infinity. We will show the non-existence of growing modes at the left-hand side of the complex-plane for large $\lambda$ (so, since the eigenvalues of $\mathcal{A}^{\lambda}$ appear in conjugate pairs, there are not growing modes in all for large $\lambda$ ).

Lemma 2.3. There exists $\Lambda>0$, such that for $\lambda>\Lambda, \mathcal{A}^{\lambda}$ has no eigenvalues in $\{z$ : $R e z \leqq 0\}$.

The proof of Lemma 2.3] is the same as that of Lemma 4.1 in [34] which works still for $\alpha \in(0,1)$. Next, we study the behavior of $\mathcal{A}^{\lambda}$ for small positive $\lambda$ and it is the more delicate part in the theory. The next two result are the heart of the Vock\&Hunziker theory for obtaining that every discrete eigenvalue of the limiting operator $\mathcal{L}_{c}=D^{\alpha}+c-\varphi_{c}$ is stable with respect to the family $\mathcal{A}^{\lambda}$ for small positive $\lambda$ (see Chapter 19 in Hislop\&Sigal [22]). The following result extends those of Lin in [34] for the case $\alpha \in(0,1)$.

Lemma 2.4. Given $F \in C_{0}^{\infty}(\mathbb{R})$. Consider any sequence $\lambda_{n} \rightarrow 0^{+}$and $\left\{u_{n}\right\} \subset H^{\alpha}(\mathbb{R})$ satisfying

$$
\left\|\mathcal{A}^{\lambda_{n}} u_{n}\right\|+\left\|u_{n}\right\| \leqq M_{1}<\infty
$$

for some constant $M_{1}$. Then if $w-\lim _{n \rightarrow \infty} u_{n}=0$, we have

$$
\lim _{n \rightarrow \infty}\left\|F u_{n}\right\|=0
$$

and

$$
\lim _{n \rightarrow \infty}\left\|\left[\mathcal{A}^{\lambda_{n}}, F\right] u_{n}\right\|=0 .
$$

Proof. The convergence in (2.18) is immediately. Next, from the relation $\mathcal{A}^{\lambda_{n}}=D^{\alpha}+c+$ $\mathcal{K}^{\lambda_{n}}$, with $\mathcal{K}^{\lambda_{n}}$ defined in (2.13), we have

$$
\left[\mathcal{A}^{\lambda_{n}}, F\right] u_{n}=\left[D^{\alpha}, F\right] u_{n}+\left[\mathcal{K}^{\lambda_{n}}, F\right] u_{n} .
$$

Now, we show that every commutator at the right-hand side of (2.20) goes to zero for $n \rightarrow \infty$.

1) $\left[D^{\alpha}, F\right] u_{n} \rightarrow 0$ as $n \rightarrow \infty$ : for $\mathcal{C}_{\alpha} \equiv\left[D^{\alpha}, F\right]$ we have that $\mathcal{C}_{\alpha}: L^{2}(\mathbb{R}) \rightarrow L^{2}(\mathbb{R})$ is a compact operator for every $\alpha \in(0,1)$. Indeed, from Theorem 3.3 in [44] we know that $\mathcal{C}_{\alpha}$ is a bounded operator on $L^{2}(\mathbb{R})$. With regard to the compactness property, we have from relation (3.18) in [44] the representation formula

$$
\mathcal{C}_{\alpha}=i k(\alpha) \int_{0}^{\infty}\left[F, P_{t}\right] t^{-\alpha} \frac{d t}{t}, \quad \alpha \in(0,1),
$$

where $P_{t}=\left(1-t^{2} \partial_{x}^{2}\right)^{-1}$ and $k(\alpha)=(2 i / \pi) \sin (\pi \alpha / 2)$. Now, since the symbol associated to the operator $P_{t}, p_{t}(\xi)=\frac{1}{1+t^{2} \xi^{2}}$, satisfies that $\frac{d}{d \xi} p_{t}(\xi) \rightarrow 0$ when $|\xi| \rightarrow$ $\infty$, by Theorem $\mathrm{C}$ in $[15]$ we obtain that the commutator $\left[F, P_{t}\right]: L^{2}(\mathbb{R}) \rightarrow L^{2}(\mathbb{R})$ is compact. Therefore $\mathcal{C}_{\alpha}$ is compact because is the limit of a sequence of compact operators. It finishes this item. 
2) $\left[\mathcal{K}^{\lambda}, F\right] u_{n} \rightarrow 0$ as $n \rightarrow \infty$ : We star for studying the commutator

$$
\left[\mathcal{E}^{\lambda} D^{\alpha}, F\right] u_{n}=\mathcal{E}^{\lambda} D^{\alpha}\left(F u_{n}\right)-F \mathcal{E}^{\lambda} D^{\alpha} u_{n}
$$

with $\mathcal{E}^{\lambda}=\frac{\lambda}{\lambda-c \partial_{x}}$. Indeed, since $F u_{n} \rightarrow 0$ on $L^{2}(\mathbb{R})$ by $(2.18)$ and $\mathcal{E}^{\lambda} D^{\alpha}: L^{2}(\mathbb{R}) \rightarrow$ $L^{2}(\mathbb{R})$ is a bounded operator for $\alpha \in(0,1)$ we have immediately that the first term of the right-hand side of $(2.22)$ goes to zero. Now, we consider $\delta>0$ such that $\delta+\alpha<1$. Then it is not difficult to see that $\left\{\mathcal{E}^{\lambda} D^{\alpha} u_{n}\right\}$ is a bounded sequence in $H^{\delta}(\mathbb{R})$. Therefore, by the local compact embedding of $H^{\delta}(\mathbb{R})$ in $L^{2}(\mathbb{R})$ and a Cantor diagonalization argument we have $\mathcal{E}^{\lambda} D^{\alpha} u_{n} \rightarrow 0$ in $L_{l o c}^{2}(\mathbb{R})$. Thus, $F \mathcal{E}^{\lambda} D^{\alpha} u_{n} \rightarrow 0$ in $L^{2}(\mathbb{R})$.

Now, we study the commutator

$$
\left[\frac{c \partial_{x}}{\lambda-c \partial_{x}} \varphi_{c}, F\right] u_{n}=\frac{c \partial_{x}}{\lambda-c \partial_{x}}\left(\varphi_{c} F u_{n}\right)-F \frac{c \partial_{x}}{\lambda-c \partial_{x}}\left(\varphi_{c} u_{n}\right)
$$

Thus, since $\varphi_{c} F u_{n} \rightarrow 0$ and $\frac{c \partial_{x}}{\lambda-c \partial_{x}} \in B\left(L^{2}(\mathbb{R})\right)$ we obtain immediately that the first term of the right-hand side of (2.23) goes to zero. Next, we consider

$$
w_{n}=\frac{c \partial_{x}}{\lambda-c \partial_{x}}\left(\varphi_{c} u_{n}\right) \equiv \mathcal{P}\left(\varphi_{c} u_{n}\right) .
$$

We shall see that $\left\{w_{n}\right\}$ is bounded in $H^{\alpha}(\mathbb{R})$. Indeed, since $\mathcal{P} \in B\left(H^{\alpha}(\mathbb{R})\right) \cap$ $B\left(L^{2}(\mathbb{R})\right)$ (since $\left.D^{\alpha \mathcal{P}}=\mathcal{P} D^{\alpha}\right)$, we obtain

$$
\begin{aligned}
\left\|w_{n}\right\|_{H^{\alpha}} & \leqq\left\|\varphi_{c} u_{n}\right\|_{H^{\alpha}} \leqq\left\|\left[D^{\alpha}, \varphi_{c}\right] u_{n}\right\|+\left\|\varphi_{c} D^{\alpha} u_{n}\right\|+\left\|\varphi_{c} u_{n}\right\| \\
& \leqq C_{0}\left\|D^{\alpha} \varphi_{c}\right\|_{\infty}\left\|u_{n}\right\|+\left\|\varphi_{c}\right\|_{\infty}\left\|u_{n}\right\|_{H^{\alpha}} \\
& \leqq C_{1}\left\|\varphi_{c}\right\|_{H^{1+\alpha}}\|\| u_{n} \|_{H^{\alpha}} \leqq M_{2},
\end{aligned}
$$

where we used Theorem 3.3 in 44 and the embedding $L^{\infty}(\mathbb{R}) \hookrightarrow B M O$. Therefore, $w_{n} \rightarrow f$ in $H^{\alpha}(\mathbb{R})$. Next, since $\mathcal{P}\left(\varphi_{c} u_{n}\right) \rightarrow 0$ in $L^{2}(\mathbb{R})$ we obtain that $f \equiv 0$. Then, by the local compact embedding $H^{\alpha}(\mathbb{R}) \hookrightarrow L^{2}(\mathbb{R})$ we obtain finally that $F w_{n} \rightarrow 0$ as $n \rightarrow \infty$. It finishes this item and the proof of the Lemma.

Remark 2.3. We note that Lemma 2.4 implies that the commutator operator $\left[\mathcal{A}^{\lambda}, F\right]$ is compact for $F \in C_{0}^{\infty}(\mathbb{R})$.

The next lemma represents a crucial piece in the asymptotic perturbation theory.

Lemma 2.5. Let $z \in \mathbb{C}$ with Rez $\leqq \frac{1}{2}$ c, then there is $n>0$ such that for all $u \in C_{0}^{\infty}(|x| \geqq$ $n)$, we have

$$
\left\|\left(\mathcal{A}^{\lambda}-z\right) u\right\| \geqq \frac{1}{4} c\|u\|
$$

when $\lambda$ is sufficiently small. 
Proof. Let $n>0$ such that $\max _{|x| \geqq n}\left|\varphi_{c}(x)\right| \leqq \frac{c}{4}$. Then for $u \in C_{0}^{\infty}(|x| \geqq n)$, it follows from the proof of Lemma 2.1 above and the bounded property of the operator $\frac{c \partial_{x}}{\lambda+c \partial_{x}}$ on $L^{2}(\mathbb{R})$, that

$$
\begin{aligned}
\operatorname{Re}\left\langle\left(\mathcal{A}^{\lambda}-z\right) u, u\right\rangle & \geqq(c-\operatorname{Re} z)\|u\|^{2}-\operatorname{Re}\left\langle u_{n} \varphi_{c}, \frac{c \partial_{x}}{\lambda+c \partial_{x}} u_{n}\right\rangle \\
& \geqq\left(c-\operatorname{Re} z-\max _{|x| \geqq n}\left|\varphi_{c}(x)\right|\right)\|u\|^{2} \geqq \frac{1}{4} c\|u\|^{2} .
\end{aligned}
$$

Then, since $\left|\operatorname{Re}\left\langle\left(\mathcal{A}^{\lambda}-z\right) u, u\right\rangle\right| \leqq\left\|\left(\mathcal{A}^{\lambda}-z\right) u\right\|\|u\|$, we finish the proof.

Thus, from Lemmas 2.4 2.5 above we can apply the asymptotic perturbation theory in [48] (see also, Definition 19.5, Theorem 19.12 and Lemmas 19.13-19.14 in Chapter 19 in Hislop\&Sigal [22]) to the continuous family of closed operators $\mathcal{A}^{\lambda}$ and to get the eigenvalue perturbations of $\mathcal{A}^{0} \equiv \mathcal{L}_{c}=D^{\alpha}+c-\varphi_{c}$ to $\mathcal{A}^{\lambda}$ with a small $\lambda$ positive. More exactly, we have the following stability result for every discrete eigenvalue of $\mathcal{L}_{c}$ with $\alpha \in(0,1)$.

Theorem 2.1. Each discrete eigenvalue $\gamma$ of $\mathcal{L}_{c}$ with $\gamma \leqq \frac{1}{2}$ c is stable with respect to the family $\mathcal{A}^{\lambda}$ in the sense that: there exists $\lambda_{1}, \delta>0$, such that for $\lambda \in\left(0, \lambda_{1}\right)$, we have

(i) $A_{\delta}(\gamma)=\{z: 0<|z-\gamma|<\delta\} \subset \Delta_{b}$, where $\Delta_{b}$ is called the region of boundeness for the family $\left\{\mathcal{A}^{\lambda}\right\}$ and defined by

$$
\Delta_{b} \equiv\left\{z: R_{\lambda}(z)=\left(\mathcal{A}^{\lambda}-z\right)^{-1} \text { exists and is uniformly bounded for } \lambda \in\left(0, \lambda_{1}\right)\right\} \text {. }
$$

(ii) Let $\Gamma$ be a simple closed curve about $\gamma$ such that $\Gamma \subset A_{\delta}(\gamma) \subset \rho\left(\mathcal{L}_{c}\right) \cap \rho\left(\mathcal{A}^{\lambda}\right)$, for all $\lambda$ small, and define the associated Riesz projector for $\mathcal{A}^{\lambda}$

$$
P_{\lambda}=-\frac{1}{2 \pi i} \oint_{\Gamma} R_{\lambda}(z) d z
$$

Then,

$$
\lim _{\lambda \rightarrow 0^{+}}\left\|P_{\lambda}-P_{\gamma}\right\|=0,
$$

where $P_{\gamma}$ is the Riesz projector for $\mathcal{L}_{c}$ and $\gamma$.

Remark 2.4. It follows from Theorem 2.1 that for all $0<\lambda \ll 1$, the operators $\mathcal{A}^{\lambda}$ have discrete spectra inside the domain determined by $\Gamma$ with total algebraic multiplicity equal to that of $\gamma$, because from (2.26) we obtain that $\operatorname{dim}\left(\operatorname{Im} P_{\lambda}\right)=\operatorname{dim}\left(\operatorname{Im} P_{\gamma}\right)$ for $\lambda$ small. In order to simplify the notation, we write $\operatorname{dim}\left(P_{\lambda}\right)$ to refer $\operatorname{dim}\left(\operatorname{Im} P_{\lambda}\right)$.

Proof. As the proof follows the same lines as that of Theorem 19.12 in [22], by convenience of the reader we give the main points of the analysis. The prove of item (i) follows from the property of $\gamma$ to be an isolated point of the spectrum of $\mathcal{L}_{c}$ and Lemma 2.5 (see Lemma 19.14 in [22]). 
About item (ii), from Proposition 2.1 and the property $\rho\left(\mathcal{L}_{c}\right) \cap \Delta_{b} \neq \emptyset$, we obtain from Kato [26] the following strong resolvent convergence

$$
\lim _{\lambda \rightarrow 0^{+}} R_{\lambda}(z) u=\left(\mathcal{L}_{c}-z\right)^{-1} u, \quad \text { for all } u \in C_{0}^{\infty}(\mathbb{R}) .
$$

Hence the Riesz projections $P_{\lambda}$ satisfies $\lim _{\lambda \rightarrow 0^{+}} P_{\lambda} u=P_{\gamma} u$, therefore we obtain from the principle of the non-expansion of the spectrum, the inequality $\operatorname{dim}\left(P_{\lambda}\right) \geq \operatorname{dim}\left(P_{\gamma}\right)$ (see Lemma 1.23 in Kato [26], pg. 438). Now, as $P_{\gamma}$ is self-adjoint we have $\lim _{\lambda \rightarrow 0^{+}} P_{\lambda}^{*} u=P_{\gamma} u$, and so, by using Lemma 1.24 in Kato [26], the two convergence of the Riesz projectors and the local compactness Lemma 2.4, we have the inequality $\operatorname{dim}\left(P_{\lambda}\right) \leq \operatorname{dim}\left(P_{\gamma}\right)$, for all $0<\lambda \ll 1$, and so the norm convergence of the projections in (2.26). It finishes the Theorem.

2.2.2. The moving kernel formula. In this subsection we study the perturbation of the eigenvalue $\gamma=0$ associated to $\mathcal{L}_{c}$ with respect to the family $\mathcal{A}^{\lambda}$ for small $\lambda>0$. For this purpose, we derive a moving kernel formula in the same spirit as in Lin [34] in order to determine when the zero eigenvalue will jump to the right or to the left. We will see that it depends of the sign of the derivative of the momentum: $\frac{d}{d c}\left\langle\varphi_{c}, \varphi_{c}\right\rangle$.

By hypotheses, we have that $\operatorname{ker}\left(\mathcal{L}_{c}\right)=\left[\frac{d}{d x} \varphi_{c}\right]$. Then we obtain that $\operatorname{dim}\left(P_{0}\right)=1$, for $P_{0}$ being the Riesz projector associated to $\gamma=0$ and $\mathcal{L}_{c}$. Therefore from Theorem 2.1 one has $\operatorname{dim}\left(P_{\lambda}\right)=1$ for all $0<\lambda \ll 1$. So, we obtain that $\mathcal{A}^{\lambda}$ has exactly one spectral point in the disc $B(0 ; \epsilon)=\{\mu \in \mathbb{C}:|\mu|<\epsilon\}$, with $\epsilon$ small, and it is non degenerate (simple). Moreover, since the eigenvalues of $\mathcal{A}^{\lambda}$ appear in conjugates pairs, we have that there is only one real eigenvalue $b_{\lambda}$ of $\mathcal{A}^{\lambda}$ inside $B(0 ; \epsilon)$. We note that from the analytic property of $\mathcal{A}^{\lambda}$ with regard to $\lambda$ and from zero being a simple eigenvalue for $\mathcal{L}_{c}$, we have that the mapping $\lambda \rightarrow b_{\lambda}$ is analytic around zero.

The idea in the next result is to determine the sign of $b_{\lambda}$, for $\lambda$ small.

Lemma 2.6. Let $c>0$. Assume that $\operatorname{ker}\left(\mathcal{L}_{c}\right)=\left[\frac{d}{d x} \varphi_{c}\right]$. For $\lambda>0$ small enough, let $b_{\lambda} \in \mathbb{R}$ be the only eigenvalue of $\mathcal{A}^{\lambda}$ near origin. Then,

$$
\lim _{\lambda \rightarrow 0^{+}} \frac{b_{\lambda}}{\lambda^{2}}=-\frac{1}{\left\|\varphi_{c}^{\prime}\right\|^{2}} \frac{d F}{d c}
$$

with $F(c)=\frac{1}{2}\left\langle\varphi_{c}, \varphi_{c}\right\rangle$. Therefore, for $\frac{d F}{d c}>0$ we obtain $b_{\lambda}<0$ and for $\frac{d F}{d c}<0$ we obtain $b_{\lambda}>0$.

At this point of our theory, we will use the existence of a smooth curve of solitary wave solutions to equation (1.6),$c \rightarrow \varphi_{c} \in H^{\alpha+1}(\mathbb{R})$ (see Remark 2.5 below for other version of the limit appearing in (2.27) ). 
Proof. As the proof follows the same lines as that of (4.7) in Lin [34], by convenience of the reader we give the main points of the analysis. From Theorem 2.1 we see that for $\lambda>0$ small enough, there exists $u_{\lambda} \in D\left(\mathcal{A}^{\lambda}\right)$, such that

$$
\int_{\mathbb{R}} \varphi_{c}(x)\left|u_{\lambda}(x)\right|^{2} d x=1
$$

and $\left(\mathcal{A}^{\lambda}-b_{\lambda}\right) u_{\lambda}=0, b_{\lambda} \in \mathbb{R}$ and $\lim _{\lambda \rightarrow 0^{+}} b_{\lambda}=0$. Next, it is not difficult to see that for $R e z \leqq \frac{c}{2}$ and $u, v$ satisfying $\left(\mathcal{A}^{\lambda}-z\right) u=v$ we have

$$
\|u\|_{H^{\frac{\alpha}{2}}} \leq M \int_{\mathbb{R}}\left|\varphi_{c}(x) \| u(x)\right|^{2} d x+\int_{\mathbb{R}}|v(x)|^{2} d x,
$$

with $M$ independent of $\lambda$. Thus, we obtaining immediately that $\left\|u_{\lambda}\right\|_{H^{\frac{\alpha}{2}}} \leq C$, for some constant $C>0$ which does not depend on $\lambda>0$. Therefore, $u_{\lambda} \rightarrow u_{0}$ in $H^{\frac{\alpha}{2}}$ as $\lambda \rightarrow 0^{+}$. It is not difficult to see that $u_{0} \neq 0$ and $\int_{\mathbb{R}} \varphi_{c}(x)\left|u_{\lambda}-u_{0}\right|^{2} d x \rightarrow 0$ as $\lambda \rightarrow 0^{+}$(because $\varphi_{c}(x) \rightarrow 0$ and the renormalization condition in (2.28) $)$. Moreover, the relation $\left(\mathcal{A}^{\lambda}-\right.$ $\left.b_{\lambda}\right)\left(u_{\lambda}-u_{0}\right)=b_{\lambda} u_{0}+\left(\mathcal{L}_{c}-\mathcal{A}^{\lambda}\right) u_{0}$, implies from (2.28) and Proposition 2.1 that $u_{\lambda} \rightarrow u_{0}$ in $H^{\frac{\alpha}{2}}$. Then , $\mathcal{A}^{\lambda} u_{\lambda} \rightarrow \mathcal{L}_{c} u_{0}=0$ as $\lambda \rightarrow 0^{+}$. By hypothesis we have $u_{0}=\theta \frac{d}{d x} \varphi_{c}=\varphi_{c}^{\prime}$ (without loss of generality we can assume $\theta=1$ ). So, $u_{\lambda} \rightarrow \varphi_{c}^{\prime}$ in $H^{\frac{\alpha}{2}}$. The relation,

$$
\frac{b_{\lambda}}{\lambda}\left\langle u_{\lambda}, \varphi_{c}^{\prime}\right\rangle=\frac{1}{c}\left\langle\left(\varphi_{c}-D^{\alpha}\right) u_{\lambda}, \frac{c \partial_{x}}{\lambda-c \partial_{x}} \varphi_{c}\right\rangle \rightarrow-\frac{1}{c}\left\langle\left(\varphi_{c}-D^{\alpha}\right) \varphi_{c}^{\prime}, \varphi_{c}\right\rangle=\left\langle\varphi_{c}^{\prime}, \varphi_{c}\right\rangle=0,
$$

implies that $\frac{b_{\lambda}}{\lambda} \rightarrow 0$ as $\lambda \rightarrow 0^{+}$.

Next, we calculate $\lim _{\lambda \rightarrow 0^{+}} \frac{b_{\lambda}}{\lambda^{2}}$. We write $u_{\lambda}=c_{\lambda} \varphi_{c}^{\prime}+\lambda v_{\lambda}$, with $c_{\lambda}=\left\langle u_{\lambda}, \varphi_{c}^{\prime}\right\rangle /\left\langle\varphi_{c}^{\prime}, \varphi_{c}^{\prime}\right\rangle$. Then, $\left\langle v_{\lambda}, \varphi_{c}^{\prime}\right\rangle=0$ and $c_{\lambda} \rightarrow 1$ as $\lambda \rightarrow 0^{+}$. Following the same strategy as in [34], we can show that $v_{\lambda} \rightarrow v_{0}$ in $H^{\frac{\alpha}{2}}$ with $v_{0} \neq 0$ and satisfying $\mathcal{L}_{c} v_{0}=\varphi_{c}$ and $\left\langle v_{0}, \varphi_{c}^{\prime}\right\rangle=0$. Since $\mathcal{L}_{c}\left(\frac{d}{d c} \varphi_{c}\right)=-\varphi_{c}$ it follows that

$$
v_{0}=-\frac{d}{d c} \varphi_{c}+d_{0} \varphi_{c}^{\prime}, \quad d_{0}=\frac{\left\langle\frac{d}{d c} \varphi_{c}, \varphi_{c}^{\prime}\right\rangle}{\left\|\varphi_{c}^{\prime}\right\|^{2}}
$$

Similarly as in [34], we can rewrite $u_{\lambda}=\overline{c_{\lambda}} \varphi_{c}^{\prime}+\lambda \overline{v_{\lambda}}$, with $\overline{c_{\lambda}} \rightarrow 1, \overline{v_{\lambda}} \rightarrow-\frac{d}{d c} \varphi_{c}$ in $H^{\frac{\alpha}{2}}$, as $\lambda \rightarrow 0^{+}$. Moreover, the equality

$$
\frac{b_{\lambda}}{\lambda^{2}}\left\langle u_{\lambda}, \varphi_{c}^{\prime}\right\rangle=-\frac{\overline{c_{\lambda}}}{c}\left\langle\frac{c \partial_{x}}{\lambda-c \partial_{x}} \varphi_{c}, \varphi_{c}\right\rangle-\frac{1}{c}\left\langle\frac{c \partial_{x}}{\lambda-c \partial_{x}}\left(\varphi_{c}-D^{\alpha}\right) v_{\lambda}, \varphi_{c}\right\rangle
$$

implies the limit

$$
\frac{b_{\lambda}}{\lambda^{2}}\left\langle u_{\lambda}, \varphi_{c}^{\prime}\right\rangle \rightarrow \frac{1}{c}\left\langle\varphi_{c}, \varphi_{c}\right\rangle-\frac{1}{c}\left\langle\left(\varphi_{c}-D^{\alpha}\right) \frac{d}{d c} \varphi_{c}, \varphi_{c}\right\rangle=-\left\langle\frac{d}{d c} \varphi_{c}, \varphi_{c}\right\rangle .
$$

Therefore,

$$
\lim _{\lambda \rightarrow 0^{+}} \frac{b_{\lambda}}{\lambda^{2}}=-\frac{1}{\left\|\varphi_{c}^{\prime}\right\|^{2}}\left\langle\frac{d}{d c} \varphi_{c}, \varphi_{c}\right\rangle .
$$

We finishes the Lemma. 
Remark 2.5. In the proof of Lemma 2.6 the existence of the curve of solitary waves $c \rightarrow \varphi_{c} \in H^{\alpha+1}(\mathbb{R})$ was used exactly for obtaining the relation $\mathcal{L}_{c}\left(\frac{d}{d c} \varphi_{c}\right)=-\varphi_{c}$. Thus, it is not difficult to see that we can change the hypothesis on the curve by the existence of $\psi \in D\left(\mathcal{L}_{c}\right)$ such that $\mathcal{L}_{c} \psi=\varphi$, with $\varphi$ being a positive solution for (1.6). Therefore, supposing that for $\mathcal{L}=D^{\alpha}+c-\varphi$ we have $\operatorname{ker}(\mathcal{L})=\left[\frac{d}{d x} \varphi\right]$, then relation (2.27) can be rewrite as

$$
\lim _{\lambda \rightarrow 0^{+}} \frac{b_{\lambda}}{\lambda^{2}}=\frac{1}{\left\|\varphi^{\prime}\right\|^{2}}\langle\psi, \varphi\rangle .
$$

Proof. [Theorem 1.2; Linear instability criterium for fKdV equations] The proof follows the same lines as in Lin 34 by using that the mapping $\lambda \in \mathcal{S} \rightarrow \mathcal{A}^{\lambda}$ represents an analytical family of operators of type- $A$, Theorem 2.1. Lemma 2.6. Proposition 2.3 and Lemma 2.3 above. So, there exists $\lambda>0$ and $0 \neq u \in H^{\alpha}(\mathbb{R})$ such that $\mathcal{A}^{\lambda} u=0$ and therefore $e^{\lambda t} u(x)$ is a purely growing mode solution to (1.31).

Proof. [Corollary 1.1: Linear instability of ground state for fKdV equations] From the analysis in the proof of Theorem [1.1, it follows that the self-adjoint operator $\mathcal{L}_{c}=D^{\alpha}+c-Q_{c}$ satisfies that $n\left(\mathcal{L}_{c}\right)=1$ and $\operatorname{Ker}\left(\mathcal{L}_{c}\right)=\left[\frac{d}{d x} Q_{c}\right]$. Now, the curve $c \rightarrow V(c)=Q_{c} \in H^{\alpha}(\mathbb{R})$ is at least of $C^{1}$-class. Indeed, we know that $Q_{c}(x)=2 c Q\left(c^{1 / \alpha} x\right)$, for $Q$ being the ground state associated to (1.10), then from the relation

$$
V^{\prime}(c)=2 Q\left(c^{1 / \alpha} x\right)+\frac{2}{\alpha} c^{1 / \alpha} x Q^{\prime}\left(c^{1 / \alpha} x\right)
$$

and $R=\alpha Q+x Q^{\prime} \in H^{\alpha+1}(\mathbb{R})$ (see proof of Theorem 1.1) we obtain $V^{\prime}(c) \in H^{\alpha+1}(\mathbb{R}) \subset$ $H^{\alpha}(\mathbb{R})$. Thus,

$$
\frac{d}{d c}\left\langle Q_{c}, Q_{c}\right\rangle=4\|Q\|^{2} \frac{d}{d c} c^{2-\frac{1}{\alpha}}=4\left(2-\frac{1}{\alpha}\right) c^{1-\frac{1}{\alpha}}\|Q\|^{2}<0
$$

where we have used that $\alpha<\frac{1}{2}$ (For $\alpha=\frac{1}{2}$, we obtain the equality $\frac{d}{d c}\left\langle Q_{c}, Q_{c}\right\rangle=0$ for all c). Hence, the condition ( $i i)$ in Theorem 1.2 can be applied and therefore we finish the proof.

Proof. [Theorem 1.3; Linear instability criterium for gfKdV equations] The proof follows the same lines as that established for the linear instability criterium for the fKdV equation. But the strategy for showing the basic Lemma 2.2 and the compactness Lemma 2.4 associated to the family of linear operators $\mathcal{V}^{\lambda}: H^{\alpha}(\mathbb{R}) \longrightarrow L^{2}(\mathbb{R})$, with $\operatorname{Re} \lambda>0$,

$$
\mathcal{V}^{\lambda} v \equiv c v+\frac{c \partial_{x}}{\lambda-c \partial_{x}}\left(\varphi_{c} v-\mathcal{M} v\right)
$$

need to be changed. Indeed, with regard to Lemma 2.2 for $\mathcal{A}^{\lambda}$ we change the relation (2.14) by

$$
\left[\mathcal{V}^{\lambda}, \zeta_{d}\right]=\left(1-\mathcal{E}^{\lambda}\right)\left[D^{\alpha}, \zeta_{d}\right]+\left[\mathcal{E}^{\lambda}, \zeta_{d}\right]\left(\varphi_{c}-\mathcal{M}\right)+\left(1-\mathcal{E}^{\lambda}\right)\left[\mathcal{M}-D^{\alpha}, \zeta_{d}\right]
$$


The estimative required for the two first term in the right-hand side of (2.34) is equal to that in (2.15)-(2.16). For the third term, we use the condition in (1.38) for $\eta(\xi)=$ $\beta(\xi)-|\xi|^{\alpha}$. Indeed, it is not difficult to see that the kernel

$$
K_{r}(x, y)=(x-y) \check{\eta}(x-y) \zeta_{d}^{\prime}(r(x-y)+y) \quad r \in[0,1],
$$

where " $\breve{\eta}$ " represents the inverse Fourier transform of $\eta$, satisfies

$$
\int_{\mathbb{R}} \int_{\mathbb{R}}\left|K_{r}(x, y)\right|^{2} d x d y=\int_{\mathbb{R}} \int_{\mathbb{R}}|x \check{\eta}(x)|^{2}\left|\zeta_{d}^{\prime}(y)\right|^{2} d x d y=\left\|\eta^{\prime}\right\|^{2}\|\| \zeta_{d}^{\prime}\left\|^{2}=\frac{1}{d}\right\| \eta^{\prime}\left\|^{2}\right\|\left\|\zeta^{\prime}\right\|^{2} .
$$

Therefore,

$$
\begin{aligned}
& \left\|\left[\mathcal{M}-D^{\alpha}, \zeta_{d}\right] u\right\|^{2} \leqq \int_{\mathbb{R}}\left|\int_{0}^{1} \int_{\mathbb{R}} u(y) K_{r}(x, y) d y d r\right|^{2} d x \\
& \leqq \int_{0}^{1} \int_{\mathbb{R}}\left|\int_{\mathbb{R}} u(y) K_{r}(x, y) d y\right|^{2} d x d r \leqq \int_{0}^{1}\|u\|^{2} \int_{\mathbb{R}} \int_{\mathbb{R}}\left|K_{r}(x, y)\right|^{2} d y d x d r \\
& \leqq \frac{1}{d}\left\|\eta^{\prime}\right\|^{2}\|\| \zeta^{\prime}\left\|^{2}\right\| u \|^{2} .
\end{aligned}
$$

Therefore, $\left\|\left(1-\mathcal{E}^{\lambda}\right)\left[\mathcal{M}-D^{\alpha}, \zeta_{d}\right] u\right\| \leqq \frac{1}{d^{1 / 2}}\left\|\eta^{\prime}\right\|\|\| \zeta^{\prime}\|\| u \|$. It finishes the estimative.

Now, with regard to Lemma 2.4 for $\mathcal{V}^{\lambda}$ we change the relation (2.20) by

$$
\left[\mathcal{V}^{\lambda_{n}}, F\right]=\left[D^{\alpha}, F\right]+\left[\mathcal{W}^{\lambda_{n}}, F\right]+\left[\mathcal{M}-D^{\alpha}, F\right] .
$$

with

$$
\mathcal{W}^{\lambda}=\frac{c \partial_{x}}{\lambda-c \partial_{x}} \varphi_{c}-\frac{\lambda}{\lambda-c \partial_{x}} \mathcal{M}: L^{2}(\mathbb{R}) \rightarrow L^{2}(\mathbb{R}) .
$$

The estimative required for the two first term in the right-hand side of (2.38) is equal to that in the proof of Lemma 2.4. For the third term, we use the condition in (1.38). Indeed, since $\eta$ is bounded and continuous over $\mathbb{R}$ and $\eta^{\prime}(x) \rightarrow 0$, as $|x| \rightarrow \infty$, (because $\eta^{\prime} \in L^{2}(\mathbb{R})$ ), follows from Theorem $\mathrm{C}$ in Cordes [15] that the commutator $\left[\mathcal{M}-D^{\alpha}, F\right]$ : $L^{2}(\mathbb{R}) \rightarrow L^{2}(\mathbb{R})$ is compact. It finishes the proof of the Theorem.

\section{3. "Stability of the Blow-UP" FOR the CRITICAl FKDV EQUATiON}

In this section we obtain information of large-time asymptotic behaviour of solutions for the critical fKdV equation:

$$
u_{t}+u u_{x}-D^{1 / 2} u_{x}=0
$$

on $\mathbb{R}$. As we saw in the last sections, the orbit generated by ground state solutions $Q_{c}$ associated to equation (1.6) for $\frac{1}{2}<\alpha<2$ and $1 \leqq p<p_{\max }(\alpha)$ are nonlinearly stable in $H^{\frac{\alpha}{2}}(\mathbb{R})$ by the flow of equation (1.1) for $p<2 \alpha$ (see Theorem 1.1), moreover, the general linear instability criterium, Theorem 1.2 , shows the linear instability of $Q_{c}$ for $\frac{1}{3}<\alpha<\frac{1}{2}$ 
and $p=1$. Now, from the proof of these later results we can see that the behavior of the solutions $Q_{c}$ for $\alpha=\frac{1}{2}$ and $p=1$ is unclear, essentially because the expression

$$
\frac{d}{d c}\left\langle Q_{c}, Q_{c}\right\rangle=4\left(2-\frac{1}{\alpha}\right) c^{1-\frac{1}{\alpha}}\|Q\|^{2}
$$

is zero exactly for $\alpha=\frac{1}{2}$.

Recently Saut\&Klein in [29] had provided a detailed numerical study pertaining to the dynamics of the fKdV model (1.1) with $0<\alpha<1$ and $p=1$. For the specific case of $\alpha=\frac{1}{2}$ and with a initial data $u_{0}$ of negative energy $\left(E\left(u_{0}\right)<0\right)$ and with a mass larger that the solitary wave mass $Q_{c}\left(\left\|Q_{c}\right\|<\left\|u_{0}\right\|\right)$ the simulations show a possible blow-up phenomenon of the associated solution (see Fig. 10 in [29]). Moreover, the peak which appears to blow-up eventually gets more and more compressed laterally, grows in hight and propagates faster with a profile of a dynamically rescaled solitary wave. Here we will show that in fact we have a kind of "stability of the blow-up" near to the possible unstable ground state solutions for equation (3.1).

The strategy for showing our "stability" result follows that used by Angulo et al. in [6] (see also Angulo [5]) for studying the critical case in the model (1.1) for $\alpha \geqq 1$, namely, $p=2 \alpha, p \in \mathbb{N}$. Thus, we consider $\mathcal{L}_{c}$ be the linear, self-adjoint, closed, unbounded operator defined on $H^{1 / 2}(\mathbb{R})$ by

$$
\mathcal{L}_{c}=D^{1 / 2}+c-Q_{c},
$$

where $Q_{c}$ is the ground-state solution associated to (1.6). Therefore, from [16] we have the following properties:

1) $\mathcal{L}_{c}$ has a single negative eigenvalue which is simple, with eigenfunction $\chi_{c}>0$, the zero eigenvalue is simple with eigenfunction $Q_{c}^{\prime}$, and the remainder of the spectrum of $\mathcal{L}_{c}$ is positive and bounded away from zero.

2) The curve $c \rightarrow Q_{c}$ is $C^{1}$ with values in $H^{\frac{3}{2}}(\mathbb{R})$.

Next, we consider the conserved energy functional $E$ in (1.16) with $\alpha=\frac{1}{2}$ and $p=1$. Therefore, from (1.7)-(1.9) we have that $E\left(Q_{c}\right)=0$. Moreover, from (1.19) follows the $a$ priori estimative

$$
\left\|D^{1 / 4} u(t)\right\|^{2}\left[1-\frac{\left\|u_{0}\right\|}{\left\|Q_{c}\right\|}\right] \leqq 2 E\left(u_{0}\right) .
$$

Thus, if we consider $E\left(u_{0}\right) \leqq 0$ then necessarily we have the condition $\left\|Q_{c}\right\| \leqq\left\|u_{0}\right\|$

Now, we introduce the auxiliary functions

$$
\psi(x, t)=\mu(t)^{-\frac{1}{2}} u\left(\mu(t)^{-1} x, t\right)
$$

where

$$
\mu(t)=\frac{\left\|D^{1 / 4} u(t)\right\|^{4}}{\left\|D^{1 / 4} Q_{c}\right\|^{4}},
$$

$\mu(0)=1$ and $0 \leqq t<t^{*}$ with $t^{*}$ the maximal time of existence of the solution of (3.1) under consideration, if the solution is global, $t^{*}=+\infty$ (see Remark 2.11). Note that 
unless $u$ is the zero-solution, $\mu(t) \in(0, \infty)$ for $0<t<t^{*}$. The normalization $\mu(0)=1$ is a temporary one made to simplify the presentation of the argument and it can be dispensed (see [6]). By using $E$ defined in (1.16), it is easy to check that the function $\psi$ verifies the identities

$$
\begin{array}{ll}
(i) & \|\psi(t)\|=\|u(t)\|=\left\|u_{0}\right\|, \\
\text { (ii }) & \left\langle\psi(t), D^{1 / 2} \psi(t)\right\rangle=\left\langle Q_{c}, D^{1 / 2} Q_{c}\right\rangle, \\
\text { (iii) } & E(\psi(t))=\frac{1}{\mu(t)^{1 / 2}} E(u(t)) .
\end{array}
$$

Since the stability considered here is with respect to form, i.e., up to translation in space, we introduce the pseudo-metric

$$
\rho_{c}\left(\psi(t), Q_{c}\right)^{2}=\inf _{r \in \mathbb{R}}\left\{\left\|D^{1 / 4} \psi(\cdot+r, t)-D^{1 / 4} Q_{c}(\cdot)\right\|^{2}+c\left\|\psi(\cdot+r, t)-Q_{c}(\cdot)\right\|^{2}\right\}
$$

on $H^{1 / 4}(\mathbb{R})$. Define the set $\mathcal{K}$ to be

$$
\mathcal{K}=\left\{u_{0}: u_{0} \in H^{s}(\mathbb{R}) \text { and } E\left(u_{0}\right) \leqq 0\right\} \subset H^{\frac{1}{4}}(\mathbb{R}), \quad s>\frac{21}{16} .
$$

We recall that the condition $s>\frac{21}{16}$ ensures that the Cauchy problem for (3.1) is local well-posedness in $H^{s}(\mathbb{R})$ (see [36]). Of course, the problem to prove well-posedness in $H^{\frac{\alpha}{2}}(\mathbb{R})$ in the general case $\alpha \in\left[\frac{1}{2}, 1\right.$ ), is still open (see Remark 2.1 above).

The next theorem is a stability result which belongs to the spatial structure of the solutions of (3.1) in the critical case.

Theorem 3.1. Let $Q_{c}$ be the ground state profile for (1.6). Then, for any $\epsilon>0$ there is a $\delta=\delta(\epsilon)>0$ such that if $u_{0} \in \mathcal{K}$ with $\rho_{c}\left(u_{0}, Q_{c}\right)<\delta$ and $u$ is the solution of (3.1) corresponding to the initial value $u_{0}$, then $u \in C\left(\left[0, t^{*}\right) ; H^{1 / 4}(\mathbb{R})\right)$ and

$$
\begin{aligned}
\inf _{r \in \mathbb{R}} & \left\{c\left\|u(\cdot, t)-\mu(t)^{\frac{1}{2}} Q_{c}(\mu(t)(\cdot-r))\right\|^{2}\right. \\
& \left.+\frac{1}{\mu(t)^{1 / 2}}\left\|D^{1 / 4} u(\cdot, t)-\mu(t)^{\frac{1}{2}} D^{1 / 4} Q_{c}(\mu(t)(\cdot-r))\right\|^{2}\right\}<\epsilon
\end{aligned}
$$

for all $t \in\left[0, t^{*}\right)$, where $t^{*}$ is the maximal existence time for the solution $u$ and $\mu$ is as in (3.6).

Proof. Suppose at the outset that $\mu(0)=1$. The proof is based on the time-dependent functional

$$
B_{t}[u]=\frac{1}{\mu(t)^{1 / 2}} E(u(t))+\frac{c}{2}\left(\frac{\|u(t)\|}{\left\|Q_{c}\right\|}\right)^{2 k}\left(\|u(t)\|^{2}-\left\|Q_{c}\right\|^{2}\right)
$$

where $k \in \mathbb{N}$ will be chosen later. From the definition of $B_{t}$, it is clear that if $u$ is a solution of (3.1) then $B_{t}[u]=B_{t}\left[u_{0}\right]$. Using (3.5), (3.6), and (3.7), we may write $B_{t}[u]$ in terms of $\psi$ thusly:

$$
\tilde{B}_{t}[\psi]=E(\psi(t))+\frac{c}{2}\left(\frac{\|\psi(t)\|}{\left\|Q_{c}\right\|}\right)^{2 k}\left(\|\psi(t)\|^{2}-\left\|Q_{c}\right\|^{2}\right)
$$


where the explicit dependence on $\mu$ disappears. As it will be argued presently, if it is established that, modulo translations, the inequalities

$$
\begin{gathered}
(i) \Delta \widetilde{B}_{t} \leqq c_{0}\left\|u_{0}-Q_{c}\right\| \quad \text { and } \\
(i i) \Delta \widetilde{B}_{t} \geqq c_{1}\left\|\psi(t)-Q_{c}\right\|_{\frac{1}{4}}^{2}-c_{2}\left\|\psi(t)-Q_{c}\right\|_{\frac{1}{4}}^{3}-\sum_{j=1}^{2 k} c_{k, j}\left\|\psi(t)-Q_{c}\right\|_{\frac{1}{4}}^{j+2},
\end{gathered}
$$

hold for $\Delta \widetilde{B}_{t}=\widetilde{B}_{t}[\psi]-\widetilde{B}_{t}\left[Q_{c}\right]$, where $c_{i}, c_{k, j}$ are fixed constants, then the result in Theorem 3.1 follows in a well-known form. Hence, attention is turned to establishing these bounds. The upper bound (3.11) is a straightforward consequence of $E\left(u_{0}\right) \leqq 0$ and $E\left(Q_{c}\right)=0$ (note that $\left\|u_{0}\right\|-\left\|Q_{c}\right\| \geqq 0$ ), where the constant $c_{0}$ depends on $\left\|Q_{c}\right\|$ (and on an upper bound for the choice of $\delta$ ). To prove (3.12), consider the perturbation of the ground state $Q_{c}$

$$
\psi(x+\gamma, t)=Q_{c}(x)+a(x, t)
$$

where $a$ is a real function and $\gamma=\gamma(t)$ minimizes the functional

$$
\Pi_{t}(\gamma)=\left\|D^{1 / 4} \psi(\cdot+\gamma, t)-D^{1 / 4} Q_{c}(\cdot)\right\|^{2}+c\left\|\psi(\cdot+\gamma, t)-Q_{c}(\cdot)\right\|^{2} .
$$

Using the representation (3.13), one calculates that

$$
\begin{aligned}
& \Delta \widetilde{B}_{t}=\widetilde{B}_{t}\left[Q_{c}+a\right]-\widetilde{B}_{t}\left[Q_{c}\right] \\
& \quad=E\left(Q_{c}+a\right)-E\left(Q_{c}\right)+\frac{\eta}{2}\left(\frac{\left\|Q_{c}+a\right\|}{\left\|Q_{c}\right\|}\right)^{2 k}\left(\left\|Q_{c}+a\right\|^{2}-\left\|Q_{c}\right\|^{2}\right) \\
& \quad \geqq \frac{1}{2}\left\langle\mathcal{L}_{c} a, a\right\rangle+\frac{2 k c}{\left\|Q_{c}\right\|^{2}}\left\langle a, Q_{c}\right\rangle^{2}-c_{2}(c)\|a\|_{\frac{1}{4}}^{3}-\sum_{j=1}^{2 k} c_{k, j}(c)\|a\|_{\frac{1}{4}}^{j+2} .
\end{aligned}
$$

The inequality in (3.14) is obtained using the definition $(3.3)$ of $\mathcal{L}_{c}$, the Cauchy-Schwartz inequality and interpolation (see (1.19) ).

A suitable lower bound on the quadratic form $\mathcal{L}_{c}$ is the next order of business. Initially, since the ground state solution $u(x, t)=Q_{c}(x-c t)$ is globally defined, we have from the continuous dependence theory for the model (3.1) in $H^{s}(\mathbb{R}), s>\frac{21}{16}$, that for $t$ in some interval of time $[0, T]$, the $\inf \Pi_{t}(\gamma)$ is attained in $\gamma=\gamma(t)$ for $t \in[0, T]$ (see Lemmas 6.26.3 in Angulo et al. [6]). Hence, using that $Q_{c}$ satisfies equation $D^{1 / 2} Q_{c}+c Q_{c}-\frac{1}{2} Q_{c}^{2}=0$ we obtain

$$
\left.\frac{d}{d r} \Pi_{t}(r)\right|_{r=\gamma}=2 \int_{\mathbb{R}}\left[c Q_{c}^{\prime}(x)+D^{1 / 2} Q_{c}^{\prime}(x)\right] a(x, t) d x=2 \int_{\mathbb{R}} Q_{c}(x) Q_{c}^{\prime}(x) a(x, t) d x,
$$

which give us the following compatibility relation on $a$, namely,

$$
\int_{\mathbb{R}} Q_{c}(x) Q_{c}^{\prime}(x) a(x, t) d x=0
$$

for all $t$ in an interval of time $[0, T]$. 
The issue of obtaining the lower bound (3.12) for the right-hand side of inequality (3.14) is addressed in the next few lemmas.

Lemma 3.1. Let $\mathcal{L}_{c}=D^{1 / 2}+c-Q_{c}$. Then there exists $\sigma<0$ such that if $\widetilde{h}=Q_{c}-$ $\sigma D^{1 / 2} Q_{c}$, then

$$
\min _{\langle f, \widetilde{h}\rangle=0,\|f\|=1}\left\langle\mathcal{L}_{c} f, f\right\rangle=0 .
$$

Proof: For any given value $\sigma$, define the function $f_{0}$ by

$$
f_{0}(x)=-\frac{1}{c} Q_{c}(x)-\frac{2+2 c \sigma}{c} x Q_{c}^{\prime}(x) .
$$

Then using the relation $D^{1 / 2}\left(x Q_{c}\right)=\frac{1}{2} D^{1 / 2} Q_{c}+x D^{1 / 2} Q_{c}^{\prime}$, we obtain that $\mathcal{L}_{c} f_{0}=Q_{c}-$ $\sigma D^{1 / 2} Q_{c}=\widetilde{h}$ and, consequently, that

$$
\left\langle f_{0}, \mathcal{L}_{c} f_{0}\right\rangle=\left\langle f_{0}, \widetilde{h}\right\rangle=\left(\left\|Q_{c}\right\|^{2}+\frac{1}{2 c}\left\|D^{1 / 4} Q_{c}\right\|^{2}\right) \sigma-\frac{1}{2}\left\|D^{1 / 4} Q_{c}\right\|^{2} \sigma^{2} .
$$

It is thus obvious that for small negative values of $\sigma$, it is possible to have both

$$
\left\langle\widetilde{h}, \chi_{c}\right\rangle=\int \chi_{c} Q_{c} d x-\sigma \int \chi_{c} D^{1 / 2} Q_{c} d x \neq 0
$$

and

$$
\left\langle\mathcal{L}_{c}^{-1} \widetilde{h}, \widetilde{h}\right\rangle=\left\langle f_{0}, \widetilde{h}\right\rangle<0 .
$$

Since $\operatorname{Ker}\left(\mathcal{L}_{c}\right)=\left[Q_{c}^{\prime}\right]$ and $\widetilde{h} \in\left(\operatorname{Ker}\left(\mathcal{L}_{c}\right)\right)^{\perp}$, it follows from Weinstein [50]) (see also Lemma 6.4 in [5]) that

$$
\theta=\min \left\{\left\langle\mathcal{L}_{c} f, f\right\rangle:\|f\|=1 \text { and }\langle f, \widetilde{h}\rangle=0\right\}=0 .
$$

The proof of the existence of the minimum in (3.17) follows the same ideas as in Lemma 6.7 in Angulo et al. [6]. This completes the proof of the lemma.

Lemma 3.2. If $\widetilde{h} \equiv Q_{c}-\sigma D^{1 / 2} Q_{c}$ with $\sigma<0$ chosen as in the last lemma, then

$$
\inf \left\{\left\langle\mathcal{L}_{c} f, f\right\rangle:\|f\|=1,\langle f, \widetilde{h}\rangle=0, f \perp Q_{c} Q_{c}^{\prime}\right\} \equiv \nu>0 .
$$

Proof. Because of Lemma 3.1 , it follows $\nu \geqq 0$. Suppose that $\nu=0$. Then, we can guarantee the existence of a function $f^{*}$ such that $\left\|f^{*}\right\|=1,\left\langle f^{*}, \widetilde{h}\right\rangle=0,\left\langle f^{*}, Q_{c} Q_{c}^{\prime}\right\rangle=0$ and $\left\langle\mathcal{L}_{c} f^{*}, f^{*}\right\rangle=0$. Therefore, there exists at least one non-trivial critical point $\left(f^{*}, \tau, \theta, \nu\right)$ for the Lagrange multiplier problem

$$
\left\{\begin{array}{l}
\mathcal{L}_{c} f=\tau f+\theta \widetilde{h}+\nu Q_{c} Q_{c}^{\prime} \\
\text { subject to } \\
\|f\|=1,\left\langle f, Q_{c} Q_{c}^{\prime},\right\rangle=0 \text { and }\langle\mathrm{f}, \widetilde{\mathrm{h}}\rangle=0
\end{array}\right.
$$


Using the fact $\left\langle\mathcal{L}_{c} f^{*}, f^{*}\right\rangle=0$, it is easily seen that (3.19) implies $\tau=0$. Moreover, since $\mathcal{L}_{c} Q_{c}^{\prime}=0$, we have that $\left\langle\mathcal{L}_{c} f^{*}, Q_{c}^{\prime}\right\rangle=\left\langle f^{*}, \mathcal{L}_{c} Q_{c}^{\prime}\right\rangle=\nu \int\left(Q_{c}^{\prime}\right)^{2} Q_{c} d x=0$, which implies $\nu=0$. It is thereby concluded that

$$
\mathcal{L}_{c} f=\theta \widetilde{h}
$$

has nontrivial solutions $\left(f^{*}, \theta\right)$ satisfying the constraints. But if $f$ is the auxiliary function arising in the proof of Lemma 3.1, we have that $\mathcal{L}_{c} f_{0}=\widetilde{h}$ and so $\mathcal{L}_{c}\left(f^{*}-\theta f_{0}\right)=0$. Then $f^{*}-\theta f_{0} \in \operatorname{Ker}\left(\mathcal{L}_{c}\right)$. It follows from (3.16) that $\left\langle f_{0}, \widetilde{h}\right\rangle \neq 0$, and so $\theta=0$. Therefore, for some non-zero $\lambda \in \mathbb{R}$, it is true that $f^{*}=\lambda Q_{c}^{\prime}$, which is a contradiction since such a function cannot be orthogonal to $Q_{c} Q_{c}^{\prime}$. Therefore, the minimum in (3.18) is positive and the proof of the Lemma is completed.

We note that from (3.18) and from the specific form of $\mathcal{L}_{c}$, we have that if $f \in H^{\frac{1}{4}}(\mathbb{R})$ satisfies $\langle f, \widetilde{h}\rangle=0$ and $\left\langle f, Q_{c} Q_{c}^{\prime}\right\rangle=0$, then

$$
\left\langle\mathcal{L}_{c} f, f\right\rangle=\int\left|D^{1 / 4} f(x)\right|^{2}+\left(c-Q_{c}(x)\right)|f(x)|^{2} d x \geqq \beta_{0}\|f\|_{\frac{1}{4}}^{2}, \quad \beta_{0}>0 .
$$

Continuation of proof of Theorem 3.1 Attention is now turned to estimating the term $\frac{1}{2}\left\langle\mathcal{L}_{c} a, a\right\rangle+\frac{2 k c}{\left\|Q_{c}\right\|^{2}}\left\langle a, Q_{c}\right\rangle^{2}$ in (3.14), where $a$ satisfies the compatibility relation (3.15). We continue to carry over the notation from Lemma 3.1 and Lemma 3.2. In particular, $\sigma$ is chosen so that the conclusions of Lemma 3.1 are valid. Define $a_{\|}$and $a_{\perp}$ to be

$$
a_{\|}=\frac{\langle a, \widetilde{h}\rangle}{\|\widetilde{h}\|^{2}} \widetilde{h} \quad \text { and } \quad \mathrm{a}_{\perp}=\mathrm{a}-\mathrm{a}_{\|} .
$$

It follows from the properties of $a$ and $\widetilde{h}=Q_{c}-\sigma D^{1 / 2} Q_{c}$ that $\left\langle a_{\perp}, \widetilde{h}\right\rangle=0, \int Q_{c} Q_{c}^{\prime} a_{\perp} d x=$ 0 . Without loss of generality, take $\langle a, \widetilde{h}\rangle<0$. Thus, from Lemma 3.2, the Cauchy-Schwarz inequality and from the properties of $a, a_{\perp}, a_{\|}$and $\widetilde{h}$, it follows that

$$
\left\{\begin{array}{l}
\left\langle\mathcal{L}_{c} a_{\perp}, a_{\perp}\right\rangle \geqq D_{1}\left\|a_{\perp}\right\|^{2}, \quad\left\langle\mathcal{L}_{c} a_{\|}, a_{\|}\right\rangle=\frac{\left\|a_{\|}\right\|^{2}}{\|\widetilde{h}\|^{2}}\left\langle\widetilde{h}, \mathcal{L}_{c} \widetilde{h}\right\rangle, \\
\left\langle\mathcal{L}_{c} a_{\|}, a_{\perp}\right\rangle=\frac{\langle a, \widetilde{h}\rangle}{\|\widetilde{h}\|^{2}}\left\langle\mathcal{L}_{c} \widetilde{h}, a_{\perp}\right\rangle \geqq-D_{2}\left\|a_{\perp}\right\|\left\|a_{\|}\right\|
\end{array}\right.
$$

for some positive constants $D_{1}$ and $D_{2}$. Identity $(i i)$ in (3.7) implies $-2\left\langle a, D^{\beta} Q_{c}\right\rangle=$ $\left\|D^{\beta / 2} a\right\|^{2}$. Thus, from the Cauchy-Schwarz inequality we obtain (remember, $\sigma$ and $\langle a, \widetilde{h}\rangle$ are both negative)

$$
\begin{aligned}
\frac{2 k c}{\left\|Q_{c}\right\|^{2}}\left\langle a, Q_{c}\right\rangle^{2} & \geqq \frac{2 k c}{\left\|Q_{c}\right\|^{2}}\left(\langle a, \widetilde{h}\rangle^{2}-\sigma\langle a, \widetilde{h}\rangle\left\|D^{1 / 4} a\right\|^{2}\right) \\
& \geqq \frac{2 k c}{\left\|Q_{c}\right\|^{2}}\|\widetilde{h}\|^{2}\left\|a_{\|}\right\|^{2}+2 k c \sigma D_{3}\|a\|_{\frac{1}{4}}^{3}
\end{aligned}
$$


with $D_{3}>0$. We choose $\theta>0$ so that $D_{1}-\theta D_{2} \equiv D_{4}>0$. By Young's inequality, $\left\|a_{\perp}\right\|\left\|a_{\|}\right\| \leqq \theta\left\|a_{\perp}\right\|^{2}+\frac{1}{\theta}\left\|a_{\|}\right\|^{2}$. Finally, fix $k$ in such a way that

$$
\frac{2 k c}{\left\|Q_{c}\right\|^{2}}\|\widetilde{h}\|^{2}+\frac{\left\langle\widetilde{h}, \mathcal{L}_{c} \widetilde{h}\right\rangle}{\|\widetilde{h}\|^{2}}-\frac{D_{2}}{\theta} \equiv D_{5}>0 .
$$

With these choices, it follows from (3.21) and (3.22) that

$$
\begin{aligned}
\frac{1}{2}\left\langle\mathcal{L}_{c} a, a\right\rangle+\frac{2 k c}{\left\|Q_{c}\right\|^{2}}\left\langle a, Q_{c}\right\rangle^{2} & \geqq D_{5}\left\|a_{\|}\right\|^{2}+D_{4}\left\|a_{\perp}\right\|^{2}+2 k c \sigma D_{3}\|a\|_{\frac{1}{4}}^{3} \\
& \geqq D^{\prime}\|a\|^{2}-D^{\prime \prime}\|a\|_{\frac{1}{4}}^{3}
\end{aligned}
$$

for some positive constants $D^{\prime}$ and $D^{\prime \prime}$. With (3.23) in hand, it follows easily from the specific form of the operator $\mathcal{L}_{c}($ see $(3.20)$ ) that

$$
\frac{1}{2}\left\langle\mathcal{L}_{c} a, a\right\rangle+\frac{2 k c}{\left\|Q_{c}\right\|^{2}}\left\langle a, Q_{c}\right\rangle^{2} \geqq \widetilde{D}_{1}\|a\|_{\frac{1}{4}}^{2}-\widetilde{D}_{2}\|a\|_{\frac{1}{4}}^{3},
$$

with $\widetilde{D}_{1}, \widetilde{D}_{2}>0$. Finally, using (3.24) in conjunction with (3.14), we obtain

$$
\begin{aligned}
\Delta \widetilde{B}_{t} & \geqq \widetilde{D}_{1}\|a\|_{\frac{1}{4}}^{2}-\widetilde{D}_{2}\|a\|_{\frac{1}{4}}^{3}-c_{2}(c)\|a\|_{\frac{1}{4}}^{3}-\sum_{j=1}^{2 k} c_{k, j}(c)\|a\|_{\frac{1}{4}}^{j+2} \\
& \geqq c_{0}\|a\|_{\frac{1}{4}}^{2}-c_{1}\|a\|_{\frac{1}{4}}^{3}-\sum_{j=1}^{2 k} c_{k, j}\|a\|_{\frac{1}{4}}^{j+2}
\end{aligned}
$$

where $c_{0}, c_{1}, c_{k, j}$ are positive constants which depend only on $c$.

Now we are in position to finish Theorem 3.1. Suppose first that $u_{0}$ lies in the set $\mathcal{K}$ of "nonpositive-energy' initial values and suppose $\left\|u_{0}-Q_{c}\right\|_{\frac{1}{4}}=\delta$. Then at least for $t \in[0, T]$, it follows from (3.11) and (3.12) that

$$
q\left(\rho_{c}\left(\psi(t), Q_{c}\right)\right) \leqq \Delta \widetilde{B}_{t} \leqq c_{0} \delta
$$

where $q(x)=c_{0} x^{2}-c_{1} x^{3}-\sum_{j=1}^{2 k} c_{k, j} x^{j+2}$. Since $\|a(t)\|_{\frac{1}{4}}^{2}=\rho_{c}\left(\psi(t), Q_{c}\right)^{2}$ is a continuous function of $t \in\left[0, t^{*}\right)$, it follows from the inequality

$$
q\left(\rho_{c}\left(\psi(0), Q_{c}\right)\right) \leqq c_{0} \delta
$$

and (3.25), that given $\epsilon>0$, then for all $t \in[0, T]$,

$$
\rho_{c}\left(\psi(t), Q_{c}\right) \leqq \epsilon
$$

provided that $\delta$ is chosen small enough at the outset. To finish the proof, we need to show that inequality (3.27) is still true for $t \in\left[0, t^{*}\right)$. This part is shown using a method similar to that of the proof of Theorem 6.1 in Angulo et al. [6]. Therefore, the stability in Theorem 3.1 is established if $\mu(0)=1$. The general case, wherein the initial data is 
not necessarily such that $\mu(0)=1$ requires a little more of work, and therefore we refer the reader to see the reference [6]. This completes the proof of Theorem 3.1 .

3.1. Behaviour of the stability parameters for the critical-fKdV equation. In the proof of Theorem 3.1, we use that there is a specific choice of the translation parameter $\gamma=\gamma(t)$ such that

$$
\left\|D^{1 / 4} \psi(\cdot+\gamma, t)-D^{1 / 4} Q_{c}(\cdot)\right\|^{2}+c\left\|\psi(\cdot+\gamma, t)-Q_{c}(\cdot)\right\|^{2}=\rho_{c}\left(\psi(t), Q_{c}\right)^{2} \leqq \epsilon
$$

for all $t<t^{*}$, where $\psi$ is the rescaled version of the solution $u$ of (3.1) defined in (3.5). Moreover, a choice of $\gamma$ for which (3.28) holds may be determined via the orthogonality condition in (3.15). By an application of the implicit-function theorem as in Lemma 4.2 in [6], it is obtained that as long as $\psi$ satisfies (3.28), there is a unique, continuously differentiable choice of the value $\gamma(t)$ that achieves (3.15) provided that the initial data $u_{0} \in H^{s}(\mathbb{R})$ for $s$ sufficiently large and the profile $Q_{c} \in H^{n}(\mathbb{R})$ for $n$ large (at least for $n \geqq 3$ ). Moreover, with the hypothesis of sufficiently regularity for the initial data $u_{0}$ we can see that $\mu$ defined in (3.6) belongs to the class $C^{1}\left(\left[0, t^{*}\right): \mathbb{R}\right)$.

Thus, by following the line of argumentation in Lemma 4.3 in [6], we obtain the relation between the translation and dilation parameters involved in our stability result in Theorem 3.1.

Theorem 3.2. Let $Q_{c}$ be the ground state profile for (3.1) such that $Q_{c} \in H^{n}(\mathbb{R}), n \geqq 3$. Then, for any $\epsilon>0$ there is a $\delta=\delta(\epsilon)>0$ such that if $u_{0} \in H^{s}(\mathbb{R}) \cap \mathcal{K}$, with s sufficiently large and $\left\|u_{0}-Q_{c}\right\|_{\frac{1}{4}}<\delta$, then there exists a $C^{1}$-mapping $\gamma:\left[0, t^{*}\right) \rightarrow \mathbb{R}$ such that

i) $\left\|\psi(\cdot+\gamma(t), t)-Q_{c}\right\|_{\frac{1}{4}} \leqq \epsilon \quad$ for $t \in\left[0, t^{*}\right)$,

ii) for all $t \in\left[0, t^{*}\right)$,

$$
\left|\gamma(t)-c \mu(t) \int_{0}^{t} \sqrt{\mu(s)} d s\right| \leqq C \epsilon \mu(t)\left(\int_{0}^{t} \sqrt{\mu(s)} d s+\int_{0}^{t} \frac{\left|\mu^{\prime}(s)\right|}{\mu^{2}(s)} d s\right)
$$

where $C$ depends only on $Q_{c}$.

Remark 3.1. The statement in Theorem 3.2 deserves to be clarified at least in some points and its relation with the nonlinear stability result established in Theorem 1.1 above for the case $\alpha \in\left(\frac{1}{2}, 2\right), p<p_{\max }(\alpha)$ and $p<2 \alpha$.

(1) The regularity required on the initial data $u_{0}$ is given to ensure that the associated solution u satisfies in a classical sense the equation (3.1).

(2) An similar analysis may be made for obtaining the behavior of the parameter of translation involved in the nonlinear stability result in Theorem 1.1. In this case, $\mu(t) \equiv 1$ for all $t$ and so for $\gamma=\gamma(t)$ such that

$$
\left\|u(\cdot+\gamma, t)-Q_{c}\right\|_{\frac{\alpha}{2}} \leqq \epsilon
$$


satisfies for all $t \in\left[0, t^{*}\right)$,

$$
|\gamma(t)-c t| \leqq C \epsilon t
$$

where $C$ depends only on $Q_{c}$.

\section{NONLINEAR STABILITy AND LINEAR INSTABILITy FOR THE FBBM EQUATION}

This section is devoted to the fractional BBM equation

$$
u_{t}+u_{x}+\partial_{x}\left(u^{2}\right)+D^{\alpha} u_{t}=0
$$

for $\alpha \in\left(\frac{1}{3}, 1\right)$. As the structure of the analysis is similar to that used for the fKdV, we will only indicate the new basic differences.

Consider a solitary wave solution $u(x, t)=\psi_{c}(x-c t), c>1$, of the fBBM equation (4.1). Then the profile $\psi_{c}$ satisfies the equation

$$
D^{\alpha} \psi_{c}+\left(1-\frac{1}{c}\right) \psi_{c}-\frac{1}{c} \psi_{c}^{2}=0 .
$$

Therefore, we obtain the following Pohozaev identity

$$
(3 \alpha-1) \int_{\mathbb{R}}\left|D^{\alpha / 2} \psi_{c}\right|^{2} d x=\left(1-\frac{1}{c}\right) \int_{\mathbb{R}}\left|\psi_{c}\right|^{2} d x,
$$

proving that no finite energy solitary waves exist when $c>1$ and $\alpha \leqq \frac{1}{3}$ hold.

By considering the new variable $v(x, t)=u(x+c t, t)-\psi_{c}(x)$, it follows from (4.1) that

$$
\left(\partial_{t}-c \partial_{x}\right)\left(v+D^{\alpha} v\right)+\partial_{x}\left(v+2 \psi_{c} v+O\left(\|v\|^{2}\right)\right)=0 .
$$

The equation

$$
\left(\partial_{t}-c \partial_{x}\right)\left(v+D^{\alpha} v\right)+\partial_{x}\left(2 \psi_{c} v+v\right)=0,
$$

represents the linearized equation for (4.1) around of $\psi_{c}$. So, we will give sufficient conditions for obtaining that the solution $v \equiv 0$ is unstable by the linear flow of (4.5). More exactly, we are interested to find a growing mode solution of (4.5) with the form $v(x, t)=e^{\lambda t} u(x)$ and $\operatorname{Re} \lambda>0$. Thus, we obtain that $u$ satisfies the following non-local differential equation,

$$
D^{\alpha} u+u+\frac{\partial_{x}}{\lambda-c \partial_{x}}\left(u+2 \psi_{c} u\right)=0
$$

This motivates us to define the following family of closed linear operators $\mathcal{B}^{\lambda}: H^{\alpha}(\mathbb{R}) \longrightarrow$ $L^{2}(\mathbb{R}), \operatorname{Re} \lambda>0$, given by

$$
\mathcal{B}^{\lambda} v \equiv\left(D^{\alpha}+1\right) v+\frac{\partial_{x}}{\lambda-c \partial_{x}}\left(v+2 \psi_{c} v\right) .
$$

Next, we consider the unbounded self-adjoint operator $\mathcal{L}_{0}: H^{\alpha}(\mathbb{R}) \longrightarrow L^{2}(\mathbb{R})$ associated to (4.2)

$$
\mathcal{L}_{0}=D^{\alpha}+\left(1-\frac{1}{c}\right)-\frac{2}{c} \psi_{c}
$$


and so $\psi_{c}^{\prime} \in \operatorname{Ker}\left(\mathcal{L}_{0}\right)$.

Our first result is about the behavior of $\mathcal{B}^{\lambda}$ by depending of $\lambda$.

Proposition 4.1. For $\lambda>0$, the operator $\mathcal{B}^{\lambda}$ converges to $\mathcal{L}_{0}$ strongly in $L^{2}(\mathbb{R})$ when $\lambda \rightarrow 0^{+}$, and converges to $D^{\alpha}+1$ strongly in $L^{2}(\mathbb{R})$ when $\lambda \rightarrow \infty$.

Proof. Similar to that of Proposition 2.1.

Next, we localized the essential spectrum of $\mathcal{B}^{\lambda}, \sigma_{\text {ess }}\left(\mathcal{B}^{\lambda}\right)$.

Proposition 4.2. For any $\lambda>0$, we have

$$
\sigma_{e s s}\left(\mathcal{B}^{\lambda}\right) \subset\left\{z: R e z \geqq \frac{1}{2}\left(1-\frac{1}{c}\right)\right\} .
$$

The idea of the proof of Proposition 2.3 is the same of Proposition 1 in Lin [34]. The next lemma is similar to Lemma 2.2 above.

Lemma 4.1. Given $\lambda>0$. Let $\zeta \in C_{0}^{\infty}(\mathbb{R})$ be a cut-off function such that $\left.\zeta\right|_{\left\{|x| \leqq R_{0}\right\}}=1$, for some $R_{0}$. Define $\zeta_{d}(x)=\zeta(x / d), d>0$. Then, for each $d$, the operator $\zeta_{d}\left(\mathcal{B}^{\lambda}-z\right)^{-1}$ is compact for some $z \in \rho\left(\mathcal{B}^{\lambda}\right)$, and there exists $C(d) \rightarrow 0$ as $d \rightarrow \infty$ such that for any $u \in C_{0}^{\infty}(\mathbb{R})$,

$$
\left\|\left[\mathcal{B}^{\lambda}, \zeta_{d}\right] u\right\| \leqq C(d)\left(\left\|\mathcal{B}^{\lambda} u\right\|+\|u\|\right) .
$$

Next, we study the behavior of $\mathcal{B}^{\lambda}$ near infinity. The next result shows the non-existence of growing modes at the left-hand side of the complex-plane for large $\lambda$ (see Lin [34]), so, since the eigenvalues of $\mathcal{B}^{\lambda}$ appear in conjugate pairs, there are not growing modes in all for large $\lambda$.

Lemma 4.2. There exists $\Lambda>0$, such that for $\lambda>\Lambda, \mathcal{B}^{\lambda}$ has no eigenvalues in $\{z$ : $R e z \leqq 0\}$.

Next, we study the behavior of $\mathcal{B}^{\lambda}$ for small positive $\lambda$. It result extends those of Lin in [34] for the case of the fBBM equation (4.1).

Lemma 4.3. Given $F \in C_{0}^{\infty}(\mathbb{R})$. Consider any sequence $\lambda_{n} \rightarrow 0^{+}$and $\left\{u_{n}\right\} \subset H^{\alpha}(\mathbb{R})$ satisfying

$$
\left\|\mathcal{B}^{\lambda_{n}} u_{n}\right\|+\left\|u_{n}\right\| \leqq M_{1}<\infty
$$

for some constant $M_{1}$. Then if $w-\lim _{n \rightarrow \infty} u_{n}=0$, we have

$$
\lim _{n \rightarrow \infty}\left\|F u_{n}\right\|=0
$$

and

$$
\lim _{n \rightarrow \infty}\left\|\left[\mathcal{B}^{\lambda_{n}}, F\right] u_{n}\right\|=0
$$


Proof. The proof in this case is immediate. First, $\left[\mathcal{B}^{\lambda}, F\right]=\left[D^{\alpha}, F\right]+\left[\mathcal{G}^{\lambda}, F\right]$ with

$$
\mathcal{G}^{\lambda}=\frac{\partial_{x}}{\lambda-c \partial_{x}}\left(1+2 \psi_{c}\right)
$$

Now, from the proof of Lemma 2.4 above we have that $\left[D^{\alpha}, F\right]$ is a compact operator. Next, the convergence

$$
\left[\mathcal{G}^{\lambda_{n}}, F\right] u_{n} \rightarrow 0, \quad \text { as } n \rightarrow \infty,
$$

is proved by following the same ideas in proof of Lemma 2.5 in [34]. It finishes the Lemma.

The next lemma is basic in the stability theory (see also Chapter 19 in Hislop\&Sigal [22]), and its proof follows from the estimative

$$
\operatorname{Re}\left\langle\left(\mathcal{B}^{\lambda}-z\right) u, u\right\rangle \geqq \frac{1}{4}\left(1-\frac{1}{c}\right)\|u\|^{2} .
$$

Lemma 4.4. Let $z \in \mathbb{C}$ with Rez $\leqq \frac{1}{2}\left(1-\frac{1}{c}\right)$, then there is $n>0$ such that for all $u \in C_{0}^{\infty}(|x| \geqq n)$, we have

$$
\left\|\left(\mathcal{B}^{\lambda}-z\right) u\right\| \geqq \frac{1}{4} c\|u\|
$$

when $\lambda$ is sufficiently small.

Thus, from Lemmas 4.3,4.4 above, we have the following stability result for every discrete eigenvalue of $\mathcal{L}_{0}$ with $\alpha \in(0,1)$.

Theorem 4.1. Each discrete eigenvalue $\kappa_{0}$ of $\mathcal{L}_{0}$ with $\kappa_{0} \leqq \frac{1}{2}\left(1-\frac{1}{c}\right)$ is stable with respect to the family $\mathcal{B}^{\lambda}$.

Next, we establish the moving kernel formula for the fBBM equations.

Lemma 4.5. Let $c>1$. Assume that $\operatorname{ker}\left(\mathcal{L}_{0}\right)=\left[\frac{d}{d x} \psi_{c}\right]$. For $\lambda>0$ small enough, let $\kappa_{\lambda} \in \mathbb{R}$ be the only eigenvalue of $\mathcal{B}^{\lambda}$ near origin. Then,

$$
\lim _{\lambda \rightarrow 0^{+}} \frac{\kappa_{\lambda}}{\lambda^{2}}=-\frac{1}{c} \frac{1}{\left\|\psi_{c}^{\prime}\right\|^{2}} \frac{d M}{d c}
$$

with $M(c)=\frac{1}{2}\left\langle\left(D^{\alpha}+1\right) \psi_{c}, \psi_{c}\right\rangle$. Therefore, for $\frac{d M}{d c}>0$ we obtain $\kappa_{\lambda}<0$ and for $\frac{d M}{d c}<0$ we obtain $\kappa_{\lambda}>0$.

Proof. The proof is similar to that of Lemma 2.7 in Lin [34].

The linearized instability result for the fBBM equation (4.1) is the following:

Theorem 4.2. [Linear instability criterium for fBBM equations] Let $c \rightarrow \psi_{c} \in$ $H^{\alpha+1}(\mathbb{R})$ be a smooth curve of positive solitary wave solution to equation (4.2) with $\alpha \in$ $\left(\frac{1}{3}, \frac{1}{2}\right), p=1$. The wave-speed $c$ can be considered over some nonempty interval $I, I \subset$ 
$(1,+\infty)$. We assume that the self-adjoint operator $\mathcal{L}_{0}=D^{\alpha}+\left(1-\frac{1}{c}\right)-\frac{2}{c} \psi_{c}$ with domain $D\left(\mathcal{L}_{0}\right)=H^{\alpha}(\mathbb{R})$ satisfies

$$
\operatorname{Ker}\left(\mathcal{L}_{0}\right)=\left[\frac{d}{d x} \psi_{c}\right]
$$

Denote by $n\left(\mathcal{L}_{0}\right)$ the number (counting multiplicity) of negative eigenvalues of the operator $\mathcal{L}_{0}$. Then there is a purely growing mode $e^{\lambda t} u(x)$ with $\lambda>0, u \in H^{s}(\mathbb{R})-\{0\}, s \geqq 0$, to the linearized equation (4.5) if one of the following two conditions is true:

(i) $n\left(\mathcal{L}_{0}\right)$ is even and $\frac{d}{d c} M(c)>0$.

(ii) $n\left(\mathcal{L}_{0}\right)$ is odd and $\frac{d}{d c} M(c)<0$,

where $M(c)=\frac{1}{2}\left\langle\left(D^{\alpha}+1\right) \psi_{c}, \psi_{c}\right\rangle$.

Next, we show the nonlinear stability and linear instability theorems for the fBBM established in the introduction (Theorems 1.4 1.5) for the ground state solutions associated to the equation (4.2). Before, we study the sign of the quantity $\frac{d}{d c} M(c)$.

Lemma 4.6. Let $c>1$ and $\alpha \in\left(\frac{1}{3}, 1\right)$. Then for any solution $\psi_{c}$ of (4.2) we have

$$
2 M(c)=\left[\frac{1}{3 \alpha-1} c^{\frac{1}{\alpha}-1}(c-1)^{3-\frac{1}{\alpha}}+c^{\frac{1}{\alpha}}(c-1)^{2-\frac{1}{\alpha}}\right]\|\Psi\|,
$$

where $\Psi$ satisfies $D^{\alpha} \Psi+\Psi-\Psi^{2}=0$. Thus, we obtain that

$$
\frac{d}{d c} M(c)= \begin{cases}>0 & \text { for } \alpha \in\left[\frac{1}{2}, 1\right) \text { and } c>1 \\ >0 & \text { for } \alpha \in\left(\frac{1}{3}, \frac{1}{2}\right) \text { and } c>c_{0} \\ <0 & \text { for } \alpha \in\left(\frac{1}{3}, \frac{1}{2}\right) \text { and } 1<c<c_{0} .\end{cases}
$$

where $c_{0}>1$ is the bigger positive root of the polynomial $q(c)=6 \alpha^{2} c^{2}-4 c \alpha+1-\alpha$, and it is given in (4.22) below.

Proof. Initially, suppose that $\psi_{c}$ is a solution of (4.2) with $c>1$ then for the scaling $\Psi(x)=a \psi_{c}(b x)$ with

$$
a=\frac{1}{c-1}, \quad \text { and } \quad b=\left(\frac{c}{c-1}\right)^{1 / \alpha}
$$

we obtain that the profile $\Psi$ satisfies $D^{\alpha} \Psi+\Psi-\Psi^{2}=0$. Thus, we obtain that

$$
\left(D^{\alpha}+1\right) \psi_{c}(x)=\frac{1}{a b^{\alpha}} D^{\alpha} \Psi(x / b)+\frac{1}{a} \Psi(x / b) .
$$

Therefore, from the relation $(3 \alpha-1) \int\left|D^{\alpha / 2} \Psi(x)\right|^{2} d x=\int|\Psi(x)|^{2} d x$ we obtain

$$
2 M(c)=\left[\frac{1}{3 \alpha-1} c^{\frac{1}{\alpha}-1}(c-1)^{3-\frac{1}{\alpha}}+c^{\frac{1}{\alpha}}(c-1)^{2-\frac{1}{\alpha}}\right]\|\Psi\|^{2} \equiv p(c)\|\Psi\|^{2} .
$$


Next, we determine the sign of the derivative of $p(c)$ defined in (4.20). An simple calculation shows that

$$
p^{\prime}(c)=\frac{c^{\frac{1}{\alpha}}(c-1)}{(c-1)^{\frac{1}{\alpha}}}\left[\frac{1-\alpha}{\alpha} \frac{1}{3 \alpha-1} \frac{(c-1)^{2}}{c^{2}}+\frac{2}{\alpha} \frac{c-1}{c}+\frac{2 \alpha-1}{\alpha}\right] .
$$

Thus, it follows immediate from (4.21) that for $c>1$ and $\alpha \in\left[\frac{1}{2}, 1\right)$ we have $p^{\prime}(c)>0$. Next, it is no difficult to see that $p^{\prime \prime}(c)>0$ for every $c>1$. Moreover, since $\alpha<\frac{1}{2}$ and

$$
\lim _{c \rightarrow 1^{+}} \frac{c^{\frac{1}{\alpha}}(c-1)}{(c-1)^{\frac{1}{\alpha}}}=+\infty
$$

we have $\lim _{c \rightarrow 1^{+}} p^{\prime}(c)=-\infty$. Now, for $c \rightarrow+\infty$

$$
p^{\prime}(c) \approx c\left[\frac{1-\alpha}{\alpha} \frac{1}{3 \alpha-1}+\frac{2 \alpha+1}{\alpha}\right]
$$

and for $1>\alpha>\frac{1}{3}$, we get that $\lim _{c \rightarrow+\infty} p^{\prime}(c)=+\infty$. Therefore, there is an unique point $c_{0}>1$ such that $p^{\prime}\left(c_{0}\right)=0$. Thus, we obtain for $\alpha \in\left(\frac{1}{3}, \frac{1}{2}\right)$ that $p^{\prime}(c)<0$ for $c \in\left(1, c_{0}\right)$ and $p^{\prime}(c)>0$ for $c \in\left(c_{0},+\infty\right)$.

Now, for determining $c_{0}$, we have that $p^{\prime}(c)=0$ if and only if $q(c)=6 \alpha^{2} c^{2}-4 c \alpha+1-\alpha=$ 0 . Since, $q(1)=(3 \alpha-1)(2 \alpha-1)<0$ we have that the real zeros of $q, r_{0}$ and $c_{0}$, satisfy $r_{0}<1<c_{0}$. The exact value of $c_{0}$ is given by

$$
c_{0}=\frac{2+\sqrt{2(3 \alpha-1)}}{6 \alpha} .
$$

It finishes the proof.

Proof. [Proof of Theorem 1.4 For $\alpha \in\left(\frac{1}{3}, 1\right)$, the scaling $Q(x)=a \Phi_{c}(b x)$, with $a$ and $b$ defined in (4.19), implies that the ground state $Q$ satisfies $D^{\alpha} Q+Q-Q^{2}=0$. Therefore, from [16] follows that the self-adjoint operator $\mathcal{L}=D^{\alpha}+1-2 Q$ satisfies $\operatorname{Ker}(\mathcal{L})=\left[\frac{d}{d x} Q\right]$ and $n(\mathcal{L})=1$. Thus, a similar analysis as that in the proof of Theorem 1.1 above we conclude that for $\mathcal{J}_{c}=D^{\alpha}+\left(1-\frac{1}{c}\right)-\frac{2}{c} \Phi_{c}$ satisfies $\operatorname{Ker}\left(\mathcal{J}_{c}\right)=\left[\frac{d}{d x} \Phi_{c}\right]$ and $n\left(\mathcal{J}_{c}\right)=1$.

Now, for $M(c)=\frac{1}{2}\left\langle\left(D^{\alpha}+1\right) \Psi_{c}, \Psi_{c}\right\rangle$ we have from Lemma 4.6 that $M^{\prime}(c)>0$ exactly for $\alpha \in\left[\frac{1}{2}, 1\right)$ and $c>1$, and for $\alpha \in\left(\frac{1}{3}, \frac{1}{2}\right)$ and $c>c_{0}$. Hence, from Grillakis et.al theory [19] we finish the proof.

As consequence of Theorem 4.2 we obtain the prove of Theorem 1.5 .

Proof. [Proof of Theorem 1.5] From the proof of Theorem 1.4 we have that the selfadjoint operator $\mathcal{J}_{c}=D^{\alpha}+\left(1-\frac{1}{c}\right)-\frac{2}{c} \Phi_{c}$ satisfies $\operatorname{Ker}\left(\mathcal{J}_{c}\right)=\left[\frac{d}{d x} \Phi_{c}\right]$ and $n\left(\mathcal{J}_{c}\right)=1$. Moreover, Lemma 4.6 establishes that $\frac{d}{d c} M(c)<0$ for $\alpha \in\left(\frac{1}{3}, \frac{1}{2}\right)$ and $c \in\left(1, c_{0}\right)$, where $c_{0}$ is giving in (4.22). It finishes the Theorem. 
Remark 4.1. Next we have the following observations about the stability Theorems 1.41.5.

(1) Similarly as in the case of the $f K d V$, the statement of stability in Theorem 1.4 is a conditional one, in the sense that for all $\epsilon>0$ there is a $\delta>0$ such that if $u_{0} \in H^{s}(\mathbb{R}) \cap U_{\delta}$, for $s>\frac{3}{2}-\alpha$, then $u(t) \in U_{\epsilon}$, for all $t \in\left(-T_{s}, T_{s}\right)$, where $T_{s}$ is the maximal time of existence of $u$ satisfying $u(0)=u_{0}$. We recall that the best known result of local well-posedness for the fBBM model (4.1) is given in [36] for initial data in $H^{s}(\mathbb{R}), s>\frac{3}{2}-\alpha$ and $\alpha \in(0,1)$. It which does not allow to globalize the solution using conservation laws.

(2) We recall that in [35] was showed the existence and stability of solitary waves solutions for the $f B B M$ by considering the minimization problem

$$
I_{q}=\inf \left\{\int_{\mathbb{R}} u^{2}+\left|D^{\alpha / 2} u\right|^{2} d x: u \in H^{\frac{\alpha}{2}}(\mathbb{R}) \text { and } \int_{\mathbb{R}} \frac{u^{2}}{2}+\frac{u^{3}}{3} d x=q\right\} .
$$

For $\alpha \in\left(\frac{1}{3}, \frac{1}{2}\right)$, a critical value constrain $q_{0}=q_{0}(\alpha)$ was established in such way that for $q>q_{0}$, the set of ground state solutions associated to the variational problem above will be stable in $H^{\frac{\alpha}{2}}(\mathbb{R})$. From our analysis in Lemma 4.6 and Theorem 1.4. we note that that critical value constrain $q_{0}$ can be determined explicitly in terms of the threshold value $c_{0}$ in (4.22).

(3) Our orbital stability and linear instability results in Theorem 1.4 and Theorem 1.5 for the $f B B M$ equation show a scenario similar to that known for the generalized $B B M$ equation $(G B B M)$

$$
u_{t}+u_{x}+u^{p} u_{x}-u_{x x t}=0 .
$$

Indeed, the critical exponent for the stability of solitary waves solutions for the GBMM is $p=4$, though the explanation for instability when $p \geqq 4$ is different. In fact, from SouganidisEStrauss [47] solitary waves of the GBBM of arbitrary positive velocity are stable when $p<4$ but when $p \geqq 4$ there exists $c_{*}=c_{*}(p)$ such that the solitary waves of velocity $c<c_{*}$ are unstable (nonlinearly) while those of velocity $c>c_{*}$ are nonlinear stable.

Acknowledgements: This work was done while the author was visiting the Department of Mathematics of Paris-Sud University as a visiting professor and him was supported by FAPESP (São Paulo Research Fundation/Brazil) under the process 2016/07311-0. The author would like express their thanks to the Professors Felipe Linares and Jean-Claude Saut for useful discussions and comments in the development of the work.

\section{REFERENCES}

[1] J.P. Albert, Positivity properties and stability of solitary-wave solutions of model equations for long waves, Comm. PDE. 17 (1992), 1-22. 
[2] J.P. Albert And J.L Bona, Total positivity and the stability of internal waves in fluids of finite depth, IMA J. Applied Math. 46 (1991), 1-19.

[3] J.P. Albert, J.L Bona And J.-C. Saut, Model equations for waves in stratified fluids, Proc. Royal Soc. London A, 453 (1997), 1233-1260.

[4] M.A. Alejo And C. Muñoz, Dynamics of complex-valued modified KdV solitons with applications to the stability of breathers, Anal. PDE., 8 (2015), pp. 629-674.

[5] J. Angulo, Nonlinear Dispersive Equations: Existence and Stability of Solitary and Periodic Travelling Wave Solutions, Mathematical Surveys and Monographs (SURV), 156, AMS, (2009).

[6] J. Angulo, J. Bona, F. Linares and M. Scialom, Scaling, stability and singularities for nonlinear, dispersive wave equations: the critical case, Nonlinearity, 15, (2002), pp. 759-786.

[7] J. Angulo, J.L. Bona And M. Scialom, Stability of cnoidal waves, Advances in Differential Equations 11 (2006) pp. 1321-1374.

[8] M.K. Arnesen, Existence of solitary-wave solutions to nonlocal equations, Discrete Contin. Dyn. Syst. 36, no. 7, (2016), pp. 3483-3510.

[9] T.B. Benjamin, Internal waves of permanent form in fluids of great depth, J. Fluid Mech. 29 (1967), pp. 559-592.

[10] T.B. Benjamin, The stability of solitary waves, Proc. Royal Soc. London. A 338 (1972), 153-183.

[11] T.B. Benjamin, J.L. Bona And J.J. Mahony, Model equations for long waves in nonlinear dispersive systems, Philos. Trans. Roy. Soc. London Ser. A, 272 (1972), pp. 3-47.

[12] J.L. Bona , On the stability theory of solitary waves, Proc. Roy. Soc. London. 344 (1975), $363-374$.

[13] J.L. Bona And J.-C. Saut, Dispersive blow up of solutions of generalized KdV equations, J. Differential Equ. 103 (1993), 3-57.

[14] J.L. Bona, P.E. Souganidis And W.A. Strauss, Stability and instability of solitary waves of Korteweg-de Vries type, Proc. Roy. Soc. London Ser. A 411 (1987), pp. 395-412.

[15] H.O. CorDES, On compactness of commutators of multiplications and convolutions, and boundedness of pseudodifferential operators, J. Funct. Anal. 18 (1975), pp. 115-131.

[16] R.L. Frank and E. Lenzmann, Uniqueness of non-linear ground states for fractional Laplacians in $\mathbb{R}$, Acta Math. 210 (2)(2013), pp. 261-318.

[17] J. Ginibre And G. Velo, Commutator expansions and smoothing properties of generalized Benjamin-Ono equations, Ann. Inst. Henri Poincaré Phys. Théorique, 51 (1989), pp. 221-229.

[18] J. Ginibre And G. Velo, Smoothing properties and existence of solutions for the generalized Benjamin-Ono equations, J. Differential Equations, 93 (1991), pp. 150-212.

[19] M. Grillakis, J. Shatah, and W. Strauss, Stability theory of solitary waves in the presence of symmetry I, J. Funct. Anal., 74 (1987), pp. 160-197.

[20] M. Grillakis, J. Shatah and W. Strauss, Stability theory of solitary waves in the presence of symmetry II, J. Funct. Anal., 94 (1990), pp. 308-348.

[21] S. Herr, A.D. Ionescu, C.E. Kenig And H. Koch, A para-differential renormalization technique for nonlinear dispersive equations, Comm. Partial Differential Equations 35 (10) (2010), pp. $1827-1875$.

[22] P.D. Hislop And I.M. Sigal, Introduction to spectral theory. With applications to Schrödinger operators, Springer-Verlag, NY, (1996).

[23] A.D. Ionescu And C. E. Kenig, Global well-posedness of the Benjamin-Ono equation in low reguality spaces, J. Amer. Math. Soc. 20 (2007), pp. 753-798.

[24] R.J. IoRIO, On the Cauchy problem for the Benjamin-Ono equation, Comm. PDE, 11, (1986), 1031-1081. 
[25] T. Kapitula and A. Stefanov, A Hamiltonian-Krein (instability) index theory for solitary waves to KdV-like eigenvalue problems, Studies Appl. Math. 132 (2014), pp. 183-221.

[26] T. Kato, Perturbation theory for linear Operators, Springer, Berlin, (1976).

[27] C.E. Kenig, Y. Martel And L. Robbiano, Local well-posedness and blow-up in the energy space for a class of $L^{2}$ critical dispersion generalized Benjamin-Ono equations, Ann. I.H. Poincaré-AN., 28 (2011), pp. 853-887.

[28] C.E. Kenig, G. Ponce AND L. VeGA, Well-posedness and scattering results for the generalized Korteweg-de Vries equation via the contraction principle, Comm. Pure Appl. Math. 46, (1993), 527-620.

[29] C. Klein And J-C. SAUt, A numerical approach to blow-up issues for dispersive perturbations of Burgers' equation, Phys. D 295/296 (2015), 46-65.

[30] D.J. Korteweg AND G. DE VRIES, On the change of form of long wave advancing in a retangular canal, and on a nem type of long stationary waves, Philos. Mag., 39, no. 5, (1895), pp. 422-443.

[31] T. Kubota, D. Ko And L. DoBBs, Weakly-nonlinear long internal gravity waves in stratified fluids of finite depth, J. Hydrodynamics 12 (1978) 157.

[32] D. LAnnes, Water waves: Mathematical Theory and Asymptotics, Mathematical Survey and Monographs, 188, AMS, Providence, RI, 2013.

[33] D. LAnnes AND J-C. SAut, Remarks on the full dispersion Kadomtsev-Petviashvli equation, Kinet. Relat. Models AIMS 9 no. 4, (2013), pp. 989-1009.

[34] Z. Lin, Instability of nonlinear dispersive solitary waves, J. Funct. Anal., 255 (2008), pp. 10911124.

[35] F. Linares, D. Pilod And J-C. SAUt, Remarks on the orbital stability of ground state solutions of $f K d V$ and related equations, Adv. Differential Equations 20 (2015), no. 9-10, 835-858.

[36] F. Linares, D. PILOD AND J-C. SAUt, Dispersive perturbations of Burgers and hyperbolic equations I: Local theory, SIAM J. Math. Anal. 46 (2014), no. 2, 1505-1537.

[37] O. Lopes, Nonlocal variational problems arising in long wave propagation, EASAIM: Control, optimisation and calculus of variations, 5 (2000), pp. 501-528.

[38] O. Lopes, A linearized instability result for solitary waves, Discrete and Continuous Dynamical Systems, Series A, 8 (2002), pp. 115-119.

[39] Y. MARTEl AND F. Merle, Review on blow up and asymptotic dynamics for critical and subcritical $g K d V$ equations, Noncompact problems at the intersection of geometry, analysis, and topology, Contemp. Math., 350 (2004), pp. 157-177.

[40] Y. Martel And F. Merle, Blow up in finite time and dynamics of blow up solutions for the $L^{2}$-critical generalized KdV equation, J. Amer. Math. Soc., 15 (2002), pp. 617-664.

[41] Y. Martel And F. MerLe, Instability of solitons for the critical generalized Korteweg-de Vries equation, Geom. Funct. Anal., 11 (2001), pp. 74-123.

[42] Y. Martel And D. Pilod, Construction of a minimal mass blow up solution of the modified Benjamin-Ono equation, arXiv:1605.01837, (2016).

[43] C. Muñoz, Stability of integrable and nonintegrable structures, Adv. Differential Equations,19 (9) (2014), 947-996.

[44] M.A.M. Murray, Commutators with fractional differentiation and BMO Sobolev spaces, IUMJ, 34 (1) (1985), pp. 205-215.

[45] D. Pelinosky, Spectral stability of nonlinear waves in KdV-type evolution equations, In Spectral analysis, stability, and bifurcation in modern nonlinear physical systems (eds Kirillov ON, Pelinovsky DE), pp. 377-398. Mechanical Engineering and Solid Mechanics Series. London, UK: Wiley-ISTE Ltd. (2013). 
[46] J-C. SAut, Sur qualques généralisations de l'équation de KdV I, J. Math. Pures Appl. 58 (1979), pp. 21-61.

[47] P.E. Souganidis and W.A. Strauss, Instability of a class of dispersive solitary waves, Proc. Roy. Soc. Edinburgh Sect. A 114 (3-4) (1990), 195-212.

[48] E. Vock And W. Hunziker, Stability of Schrödinger eigenvalue problems, Comm. Math. Phys., 83 (2) (1982), pp. 281-302.

[49] G.B. Whitham, Variational methods and applications to water waves, Proc. R. Soc. A 299 (1967), pp. 6-25.

[50] M.I. WeInstein, Liapunov stability of ground states of nonlinear dispersive evolution equations. Comm. Pure Appl. Math., v. 39, (1986), pp. 51-68.

[51] M.I. Weinstein, Existence and dynamic stability of solitary wave solutions of equations arising in long wave propagation, Comm. Partial Differential Equations, 12 (1987), pp. 1133-1173. 